\title{
Dense molecular gas towards W49A: a template for extragalactic starbursts?
}

\author{
H. Roberts ${ }^{1}$, F. F. S. van der Tak ${ }^{2,3}$, G. A. Fuller ${ }^{4}$, R. Plume ${ }^{5}$, and E. Bayet ${ }^{6}$ \\ 1 Astrophysics Research Centre, School of Mathematics and Physics, Queens University Belfast, Belfast, BT7 1NN, UK \\ e-mail: h.roberts@qub.ac.uk \\ 2 SRON Netherlands Institute for Space Research, PO Box 800, 9700 AV Groningen, The Netherlands \\ 3 Kapteyn Astronomical Institute, PO Box 800, 9700 AV Groningen, The Netherlands \\ 4 Jodrell Bank Centre for Astrophysics, School of Physics and Astronomy, University of Manchester, Manchester, M13 9PL, UK \\ 5 Department of Physics and Astronomy, University of Calgary, Calgary, T2N 1N4, AB, Canada \\ ${ }^{6}$ Department of Physics and Astronomy, University College London, Gower Street, London WC1E 6BT, UK
}

Received 17 May 2010 / Accepted 19 October 2010

\begin{abstract}
Context. The $\mathrm{HCN}, \mathrm{HCO}^{+}$, and $\mathrm{HNC}$ molecules are commonly used as tracers of dense star-forming gas in external galaxies, but such observations are spatially unresolved. Reliably inferring the properties of galactic nuclei and disks requires detailed studies of sources whose structure is spatially resolved.

Aims. To understand the origin of extragalactic molecular line emission, we compare the spatial distributions and abundance ratios of $\mathrm{HCN}, \mathrm{HCO}^{+}$, and $\mathrm{HNC}$ in W49A, the most massive and luminous star-forming region in the Galactic disk.

Methods. Maps of a $2^{\prime}(6.6 \mathrm{pc})$ field at $14^{\prime \prime}(0.83 \mathrm{pc})$ resolution of the $J=4-3$ transitions of $\mathrm{HCN}, \mathrm{H}^{13} \mathrm{CN}, \mathrm{HC}^{15} \mathrm{~N}, \mathrm{HCO}^{+}, \mathrm{H}^{13} \mathrm{CO}^{+}$, $\mathrm{HC}^{18} \mathrm{O}^{+}$, and $\mathrm{HNC}$ are combined with supplementary observations of the $J=5-4$ transition of DCN and the $J=3-2$ transitions of $\mathrm{HCN}$ and $\mathrm{H}^{13} \mathrm{CO}^{+}$. Most of the data are from HARP/ACSIS, with supplementary data from JCMT Receiver A and the SCUBA archive. We use maps of the integrated intensity and line-profiles to pick out regions of the source to study in more detail. We compare column densities and abundance ratios towards these regions with each other and with predictions from gas-phase chemical models. Results. The kinematics of the molecular gas in W49A appears complex, with a mixture of infall and outflow motions. Both the line profiles and comparison of the main and rarer species show that the main species are optically thick. Two "clumps" of infalling gas that we look at in more detail appear to be at $\sim 40 \mathrm{~K}$, compared to $\geq 100 \mathrm{~K}$ at the source centre, and may be $\sim 10 \times$ denser than the rest of the outer cloud. The chemical modelling suggests that the $\mathrm{HCN} / \mathrm{HNC}$ ratio probes the current gas temperature, while the $\mathrm{HCN} / \mathrm{HCO}^{+}$ratio and the deuterium fractionation were set during an earlier, colder phase of evolution.

Conclusions. The similarity in the derived physical conditions in W49A and those inferred for the molecular gas in external galaxies suggest that $\mathrm{W} 49 \mathrm{~A}$ is an appropriate analogue of an extragalactic star forming region. Our data show that the use of $\mathrm{HCN} / \mathrm{HNC} / \mathrm{HCO}^{+}$line ratios as proxies for the abundance ratios is incorrect for $\mathrm{W} 49 \mathrm{~A}$, suggesting that using these line ratios as abundance ratios in galactic nuclei is invalid too. On the other hand, our observed isotopic line ratios such as $\mathrm{H}^{13} \mathrm{CN} / \mathrm{H}^{13} \mathrm{CO}+$ approach our modeled abundance ratios quite well in W49A. Second, the 4-3 lines of $\mathrm{HCN}$ and $\mathrm{HCO}^{+}$are much better tracers of the dense star-forming gas in W49A than the 1-0 lines, confirming similar indications for galactic nuclei. Finally, our observed $\mathrm{HCN} / \mathrm{HNC}$ and $\mathrm{HCN} / \mathrm{HCO}^{+}$ratios in W49A are inconsistent with homogeneous PDR or XDR models, indicating that irradiation does not strongly affect the gas chemistry in W49A. Overall, the W49A region appears to be a useful template for starburst galaxies.
\end{abstract}

Key words. stars: formation - ISM: abundances - ISM: clouds - ISM: molecules

\section{Introduction}

In recent years, submillimeter line emission from a variety of molecules has been detected from external galaxies in the early and local Universe (Kramer et al. 2008). The first detection of $\mathrm{HCO}^{+}$at high redshift, the $J=1-0$ transition towards the Cloverleaf quasar at $z=2.56$ (Riechers et al. 2006), was quickly followed up by higher- $J \mathrm{HCN}, \mathrm{HNC}$ and $\mathrm{CN}$ lines towards the ultra-luminous quasar APM 08279+5255 at $z=3.911$ (Wagg et al. 2005; Guélin et al. 2007). Whereas low- $J$ CO lines are used to trace the total molecular gas mass in Galactic nuclei, $\mathrm{HCN}$ and $\mathrm{HCO}^{+}$lines are often used to trace the higher-density gas. This dense gas is much more closely related to star formation activity than $\mathrm{CO}$, as shown by the tighter correlation of $L(\mathrm{HCN} 1-0)$ with $L$ (far-IR) (Gao \& Solomon 2004). However, factors other than the mass of dense gas presumably affect the HCN emission, and high- $J$ CO lines may trace the star forming activity as well as or even better than HCN 1-0 (Krumholz \& Thompson 2007; Narayanan et al. 2008; Bayet et al. 2009b).

The $\mathrm{HCN} / \mathrm{HCO}^{+}$ratio is also used to trace the chemical state of the dense gas, in particular its irradiation by UV light from a starburst or by X-rays from an AGN. For example, Kohno (2005) use the $\mathrm{HCN} / \mathrm{HCO}^{+}$ratio in nearby Seyfert galaxies to distinguish between AGNs and starbursts; Greve et al. (2009) find that excited HCN transitions trace dense gas in the two starburst galaxies, Arp 220 and NGC 6240, while sub-thermally excited $\mathrm{HCO}^{+}$lines trace gas which is $10-100$ times less dense; the $\mathrm{HCN} / \mathrm{HCO}^{+}$ratios observed by Aalto et al. (2007) towards NGC 4418 suggest emission from dense, warm gas with a PDR component, but with no X-ray chemistry. Another commonly used line ratio is $\mathrm{HCN} / \mathrm{HNC}$, which is thought to trace the gas temperature (e.g., Baan et al. 2008). 


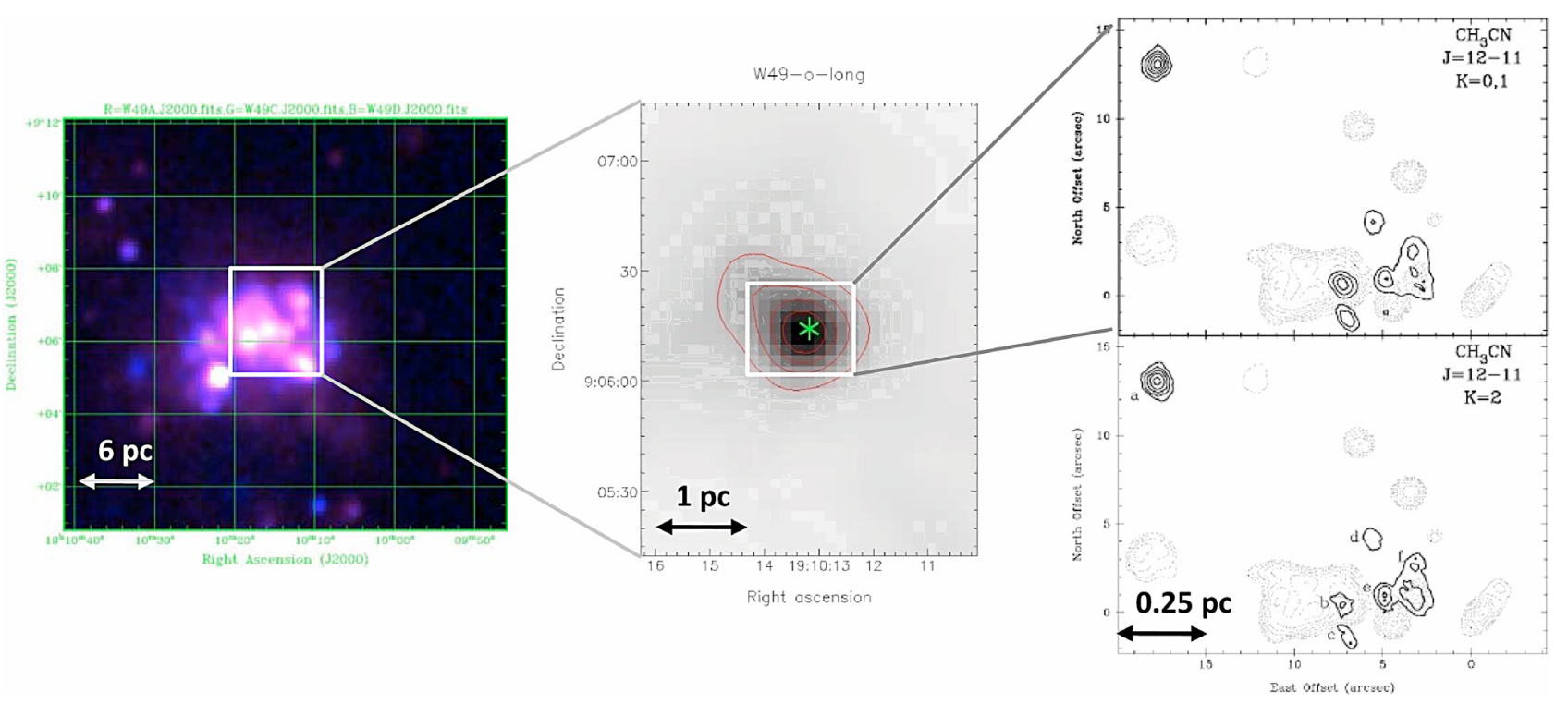

Fig. 1. Images of the W49A region at different wavelengths and spatial scales. Left: three colour MSX image of W49, showing the clustered nature of sources in the region: $8.28 \mu \mathrm{m}$ (red); $12.13 \mu \mathrm{m}$ (green); $14.65 \mu \mathrm{m}$ (blue). Centre: dust continuum emission at $850 \mu \mathrm{m}$ (SCUBA archive data; see text). Contours are at 20, 40, 60 and $80 \%$ of the peak intensity. This map is $\sim 1.5 \times 2^{\prime}$ in size, slightly smaller than our HARP maps. Right: BIMA observations of hot cores, traced by $\mathrm{CH}_{3} \mathrm{CN}$ (Wilner et al. 2001). We note that the central pixels of the HARP maps cover a cluster of several hot cores.

The angular resolution of current instrumentation at submm wavelengths ranges from $\sim 30^{\prime \prime}$ for single-dish telescopes to $\sim 1^{\prime \prime}$ for interferometers. For nearby galaxies $(D \sim 10-100 \mathrm{Mpc})$, single-dish observations thus probe scales of $1.5-15 \mathrm{kpc}$, while interferometers probe scales of 50-500 pc. In the case of highredshift galaxies, even interferometers probe size scales of $\sim \mathrm{kpc}$. The goal of this work is to improve understanding of such observations by using a local analogue. In particular, the use of line ratios to infer physical conditions depends on the assumption that the gas is roughly homogeneous on these scales, so that the lines trace the same gas. Recent authors have already questioned this assumption (Bayet et al. 2008, 2009a), and the aim of this paper is to see just how far this assumption of homogeneity is from reality, using the W49A region as a template for extragalactic regions with high star formation rates, and to explore how the observational limitations can be worked around. However, the $6.6 \mathrm{pc}$ size of the images used here falls short of the size scales resolved in distant extragalactic systems by a factor of $\gtrsim 10$.

With a luminosity in excess of $10^{7} L_{\odot}$ (Ward-Thompson \& Robson 1990) and a mass $>10^{7} M_{\odot}$, W49A is one of the most active star-forming regions in the Galactic disk, at a distance of $11.4 \mathrm{kpc}$ (Gwinn et al. 1992). The region is composed of a number of optically obscured, compact HII regions (Wilner et al. 2001) surrounded by a molecular cloud with a mass $>10^{5} M_{\odot}$ (Mufson \& Liszt 1977; Simon et al. 2001). Spectrally, W49A is extremely complex, containing numerous features contributed by W49A itself, as well as additional clouds associated with the Sagittarius spiral arm which crosses the line-of-sight twice (Plume et al. 2004). Figure 1 shows the structure of W49A at infra-red vs. sub-mm wavelengths and at spatial scales from $\sim 30$ to $<1 \mathrm{pc}$.

Welch et al. (1987) estimate the cloud density to be $>3 \times$ $10^{5} \mathrm{~cm}^{-3}$ within the inner $1 \mathrm{pc}$ (the core region) and $\sim 10^{4} \mathrm{~cm}^{-3}$ in the outer parts of the cloud. Based on observations of the CO $J=7-6$ transition, Jaffe et al. (1987) estimate kinetic temperatures of $>70 \mathrm{~K}$ in the centre, falling below $50 \mathrm{~K}$ in the outer parts of the cloud. From sub-millimetre continuum maps of the dust emission towards W49A, Ward-Thompson \& Robson (1990) find a dust temperature of $50 \mathrm{~K}$ and a dust mass $2400 M_{\odot}$ over an effective emitting region of $\sim 2$ pc. From near-infrared IRAS data they infer an additional population of small, hot $(T \sim 350 \mathrm{~K})$ dust grains or Polycyclic Aromatic Hydrocarbons (PAHs).

Before W49A can be used as an extragalactic template, a better understanding of its physical and chemical properties is needed. Rotational transitions of interstellar molecules help reaching this goal in two ways: they probe the current density, temperature, and optical depth of the gas and they measure the abundances of molecular species, which can be compared with chemical models to study the evolution of the chemical inventory and the physical conditions. Two specific tracers of the chemical state of molecular gas are the $\mathrm{HCN} / \mathrm{HNC}$ ratio and the deuteration fraction $(\mathrm{D} / \mathrm{H}$ ratio) of the gas.

The HCN/HNC abundance ratio has long been known to vary with temperature (Goldsmith et al. 1981, 1986). Although HNC and $\mathrm{HCN}$ are assumed to be formed at the same rate (through dissociative recombination of the $\mathrm{HCNH}^{+}$ion), the reactions of $\mathrm{HNC}$ with $\mathrm{O}$ and with $\mathrm{H}$, which destroy $\mathrm{HNC}$, have energy barriers which make them very slow at low temperatures. As a consequence, the $\mathrm{HCN} / \mathrm{HNC}$ ratio increases with increasing $T_{\text {kin }}$ (Schilke et al. 1992).

Observations of molecular $\mathrm{D} / \mathrm{H}$ ratios (i.e. the abundance of a chemical species where one or more $\mathrm{D}$ atoms have been substituted for the $\mathrm{H}$ atoms, relative to the abundance of the "normal" hydrogen-bearing species) also probe the kinetic temperature, as well as the chemical history, of the gas. Although the cosmic $\mathrm{D} / \mathrm{H}$ ratio is only $3 \times 10^{-5}$ (Linsky 2006), chemical fractionation concentrates deuterium in molecules at temperatures $<50$ K (Watson 1976; Millar et al. 1989). Hatchell et al. (1998) measured DCN/HCN ratios towards a number of hot molecular cores $\left(T_{\text {kin }} \geq 100 \mathrm{~K}\right)$, associated with massive protostars, and found ratios of a few times $10^{-3}$. These results, and other molecular $\mathrm{D} / \mathrm{H}$ ratios in warm gas, can be explained by assuming that the deuterium fractionation was set at temperatures $<50 \mathrm{~K}$ and preserved in the icy mantles of interstellar grains, until the 
Table 1. Summary of the observations.

\begin{tabular}{llccc}
\hline \hline Species & Transition & $\begin{array}{c}\text { Frequency } \\
\mathrm{GHz}\end{array}$ & $\begin{array}{c}t_{\text {int }}{ }^{a} \\
\mathrm{~s}\end{array}$ & $\begin{array}{c}\mathrm{rms}\left(T_{\mathrm{mb}}\right)^{b} \\
\mathrm{~K}\end{array}$ \\
\hline $\mathrm{HC}^{18} \mathrm{O}^{+}$ & $J=4-3$ & 340.631 & 540 & 0.08 \\
$\mathrm{HC}^{15} \mathrm{~N}$ & $J=4-3$ & 344.200 & 120 & 0.19 \\
$\mathrm{H}^{13} \mathrm{CN}$ & $J=4-3$ & 345.340 & 480 & 0.09 \\
$\mathrm{H}^{13} \mathrm{CO}^{+}$ & $J=4-3$ & 346.998 & 480 & 0.12 \\
$\mathrm{HCN}^{+}$ & $J=4-3$ & 354.505 & 480 & 0.07 \\
$\mathrm{HCO}^{+}$ & $J=4-3$ & 356.734 & 240 & 0.12 \\
$\mathrm{DCO}^{+}$ & $J=5-4$ & 360.170 & 480 & 0.07 \\
$\mathrm{DCN}_{\mathrm{HNC}}$ & $J=5-4$ & 362.046 & 480 & 0.06 \\
\hline $\mathrm{H}^{13} \mathrm{CO}$ & $J=3-3$ & 362.630 & 583 & 0.03 \\
$\mathrm{HCN}^{+}$ & $J=3-2$ & 260.255 & 38 & 0.22 \\
\hline
\end{tabular}

Notes. ${ }^{(a)}$ Integration time per pixel; ${ }^{(b)}$ noise level measured over the central $1^{\prime} \times 1.5^{\prime}$.

region was heated and the ices evaporated into the gas phase (see also Loinard et al. 2002; Parise et al. 2002; Vastel et al. 2003). Rodgers \& Millar (1996) show that deuterium fractionation of neutral species can survive for $10^{4}-10^{5}$ years in hot gas, post-evaporation. Deuterated molecular ions (e.g. $\mathrm{DCO}^{+}$), on the other hand, are destroyed much more rapidly when the gas is warmed to $>30 \mathrm{~K}$ (e.g. Roberts \& Millar 2007) and are not expected to be abundant in protostellar regions.

\section{Observations}

This work is based on data obtained as part of the Spectral Legacy Survey (SLS; Plume et al. 2007), being conducted at the James Clerk Maxwell Telescope (JCMT) on Mauna Kea, Hawai'i. The SLS is performing spectral imaging of $2^{\prime} \times$ $2^{\prime}$ fields towards four targets representing different star-forming environments. Once completed, the spectral range covered by the SLS will be 330-362 GHz. This paper presents observations of $\mathrm{HCN}, \mathrm{HNC} \mathrm{HCO}^{+}$, and isotopomers towards W49A (Table 1). For this data set, the "central position" refers to RA = 19:10:13.4; Dec $=$ 09:06:14 in J2000 co-ordinates.

The SLS uses the 16-pixel HARP receiver (325-375 GHz) and the ACSIS correlator (Buckle et al. 2009). The observations were carried out using the HARP4_mc jiggle position switch mode, which produces maps sampled every 7.5", and the spectra were calibrated by observing at an off-position $14^{\prime}$ to the northeast of the source. The angular resolution of the JCMT is $\sim 15^{\prime \prime}$ at $345 \mathrm{GHz}$, which is $\sim 0.8 \mathrm{pc}$ at the distance of W49. The spectral resolution of these observations is $\sim 0.8 \mathrm{~km} \mathrm{~s}^{-1}$ and the beam efficiency is 0.6 (Buckle et al. 2009). Pointing was checked every hour and the pointing is estimated to be accurate to $1.5^{\prime \prime}$.

The data were reduced using the ORAC Data Reduction pipeline (ORAC-DR). This automatically processes time-series ACSIS data, applying reduction recipes which are determined by the incoming data type, and, for HARP, outputting gridded data cubes. For SLS data, these recipes include: checking for consistency between the calculated $T_{\text {sys }}$ during the observations and the measured rms noise; checking for variations in the rms noise measured by each receptor across the map; checking for rms uniformity across the spectral range; removing baselines from every spectrum; and co-adding cubes taken at the same position and frequency. If any receptors are particularly noisy, or suffer from bad baselines, they are masked out of the time series data and not used in creating the final cubes.
Table 2. Observed line ratios.

\begin{tabular}{lcc}
\hline \hline Species & \multicolumn{2}{c}{ Ratio } \\
& $J=1-0^{a}$ & $J=4-3$ \\
\hline $\mathrm{HCN} / \mathrm{HCO}^{+}$ & 0.9 & 0.56 \\
$\mathrm{HCN} / \mathrm{HNC}$ & 1.0 & 3.7 \\
\hline Species & Ratio $J=4-3 / J=1-0$ \\
\hline $\mathrm{HCN}$ & 0.73 & \\
$\mathrm{HCO}^{+}$ & 1.25 & \\
$\mathrm{HNC}$ & 0.22 & \\
\hline
\end{tabular}

Notes. Ratios of velocity-integrated line intensities between species in the $J=1-0$ and $J=4-3$ transitions (upper table) and between the $J=$ 4-3 and $J=1-0$ transition for each of the main species (lower table). The integrated line intensities have been measured in a 67 " diameter aperture centred on the peak of the emission.

(a) IRAM $30 \mathrm{~m}$ data from Peng \& Wyrowski, priv. comm.

Selected molecular transitions have also been imaged by the SLS team using the JCMT Receiver A $(211-276 \mathrm{GHz}$; beamwidth $20^{\prime \prime}$ ). These include the $\mathrm{HCN}$ and $\mathrm{H}^{13} \mathrm{CO}^{+} J=$ 3-2 lines, which are presented below. Half-beamwidth spaced raster maps were made, covering approximately the same field of view as HARP. The $230 \mathrm{GHz}$ data were reduced and calibrated with standard Starlink procedures. Linear baselines were removed and the data were Hanning smoothed. 15 pixel $\times 15$ pixel spectral grids of the data were generated for each sight-line such that each pixel corresponds to an $(8 \times 8)^{\prime \prime}$ portion of sky.

We also made SCUBA maps of W49A, based on data from the JCMT archive. These data were reduced using the ORAC-DR package which calculates the gain from photometry calibrations and the optical depth from sky-dip measurements. The $850 \mu \mathrm{m}$ emission map is shown in Fig. 1. Finally we make use of maps of W49A in the $\mathrm{HCN}, \mathrm{HNC}$ and $\mathrm{HCO}^{+} J=$ 1-0 lines, made with the IRAM $30 \mathrm{~m}$ telescope by Peng \& Wyrowski (priv. comm.).

\section{Results}

\subsection{Spatial distributions}

Figures 2 and 3 show maps of the integrated intensities of the $\mathrm{HCN}, \mathrm{HCO}^{+}, \mathrm{HNC}, \mathrm{H}^{13} \mathrm{CN}, \mathrm{H}^{13} \mathrm{CO}^{+}$and $\mathrm{HN}^{13} \mathrm{C} J=4-3$ and the HCN $J=3-2$ lines around W49A. The same source structure, extended in the east-west direction, is seen in both the dust continuum emission (Fig. 1) and the $\mathrm{HCN}$ and $\mathrm{HCO}^{+}$lines, and also in HNC but less pronounced.

Emission from the HCN $J=3-2$ line is the most extended, being seen across most of the $(100 \times 100)^{\prime \prime}$ map. The HCN and $\mathrm{HCO}^{+} J=4-3$ emission extends over $(82.5 \times 60)^{\prime \prime}$, based on the $10 \%$ contour, and there is a slightly fainter clump of emission to the south-west, seen in both lines. The $\mathrm{H}^{13} \mathrm{CO}^{+} J=4-3$ emission extends over $(46 \times 38)^{\prime \prime}$, while $\mathrm{H}^{13} \mathrm{CN} 4-3$ is slightly more compact. The weaker isotopic lines are seen only towards the central pixels of the map: $\mathrm{HC}^{15} \mathrm{~N}$ and $\mathrm{HC}^{18} \mathrm{O} J=4-3$ are detected in a $(23 \times 23)^{\prime \prime}$ region and DCN $J=5-4$ over $(37 \times 23)^{\prime \prime}$. The $\mathrm{DCO}^{+} J=5-4$ line was not detected. All sizes refer to the $10 \%$ intensity level.

Observations of external galaxies often have limited signalto-noise and therefore consider ratios of velocity-integrated line intensities. Figure 4 shows the $\mathrm{HCN} / \mathrm{HCO}^{+}, \mathrm{H}^{13} \mathrm{CN} / \mathrm{H}^{13} \mathrm{CO}^{+}$ and $\mathrm{HCN} / \mathrm{HNC}$ ratios across the W49A map, based on the integrated intensities of the $J=4-3$ lines shown in Fig. 2, with cutoffs applied to only bring out significant structure. Table 2 gives 
A\&A 525, A107 (2011)

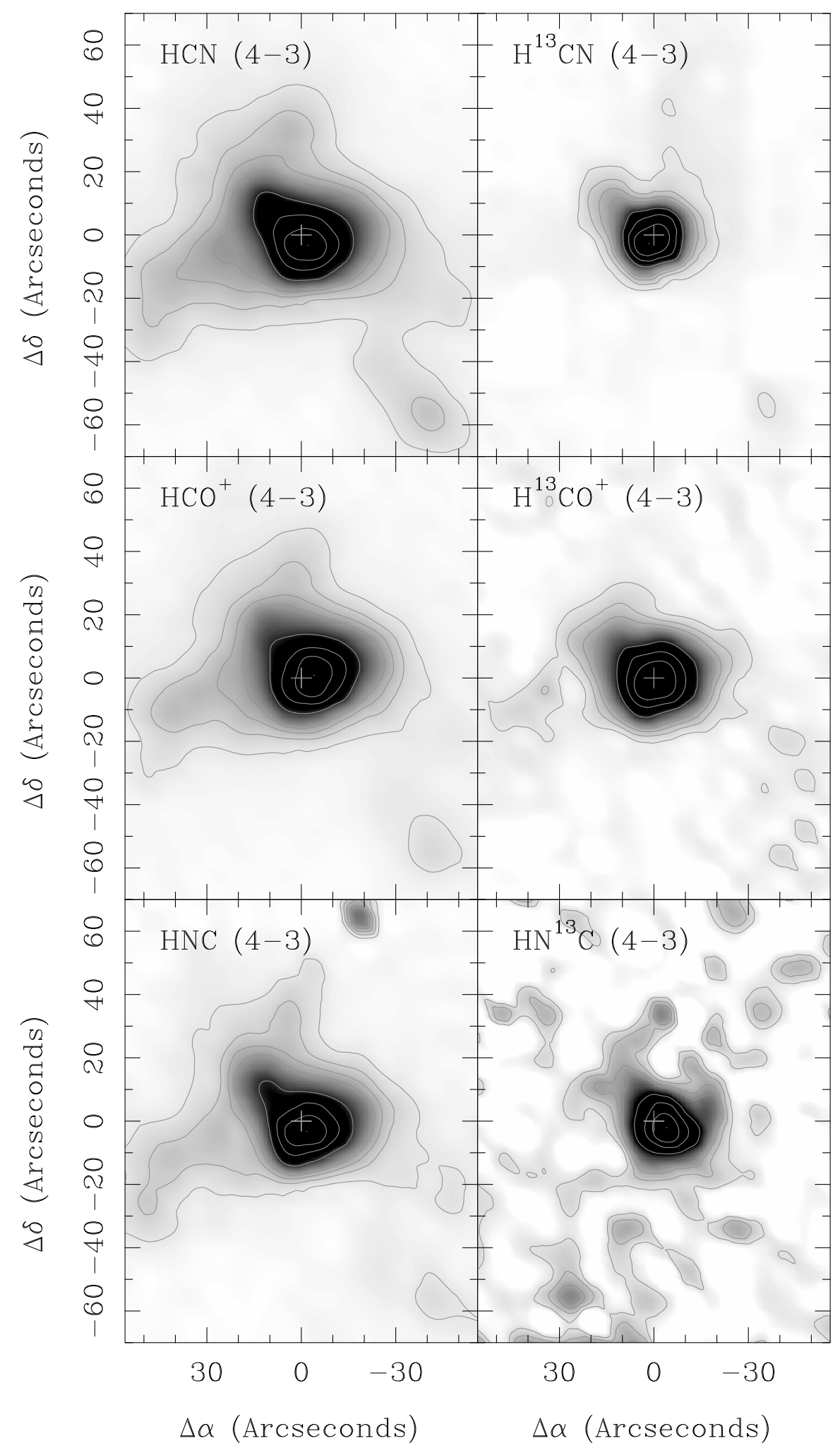

Fig. 2. Maps of the integrated intensity $\left(\int T_{\mathrm{A}}^{*} \mathrm{~d} V\right.$ ) across W49: $\mathrm{HCN} 4-3$ (top-left); $\mathrm{H}^{13} \mathrm{CN} 4-3$ (top-right); $\mathrm{HCO}^{+} 4-3\left(\right.$ middle-left); $\mathrm{H}^{13} \mathrm{CO}^{+} 4-3$ (middle-right); $\mathrm{HNC} 4-3$ (bottom-left); and $\mathrm{HN}^{13} \mathrm{C} 4-3$ (bottom-right) line intensities. The main lines are summed over $150 \mathrm{~km} \mathrm{~s}^{-1}$, while the ${ }^{13} \mathrm{C}^{-}$ substituted lines are summed over $60 \mathrm{~km} \mathrm{~s}^{-1}$. The contours show $5,10,15,20,40 \ldots 80 \%$ of the peak integrated intensity, which is $161 \mathrm{~K} \mathrm{~km} \mathrm{~s}{ }^{-1}$ for $\mathrm{HCN}, 72 \mathrm{~K} \mathrm{~km} \mathrm{~s}^{-1}$ for $\mathrm{H}^{13} \mathrm{CN}, 319 \mathrm{~K} \mathrm{~km} \mathrm{~s}^{-1}$ for $\mathrm{HCO}^{+}, 34 \mathrm{~K} \mathrm{~km} \mathrm{~s}^{-1}$ for $\mathrm{H}^{13} \mathrm{CO}^{+}, 52 \mathrm{~K} \mathrm{~km} \mathrm{~s}^{-1}$ for $\mathrm{HNC}$, and $4 \mathrm{~K} \mathrm{~km} \mathrm{~s}^{-1}$ for $\mathrm{HN}^{13} \mathrm{C}$.

the values of these ratios integrated over our maps. Over most of the map, the $\mathrm{HCN} 4-3$ line is weaker than the $\mathrm{HCO}^{+}$line, giving integrated intensity ratios $<1$. The situation is reversed for the ${ }^{13} \mathrm{C}$-substituted species: $\mathrm{H}^{13} \mathrm{CN}$ is generally stronger than $\mathrm{H}^{13} \mathrm{CO}^{+}$. This reversal indicates a high optical depth of the main isotopic $\mathrm{HCN}$ and $\mathrm{HCO}^{+}$lines, as also suggested by the 
H. Roberts et al.: Dense molecular gas towards W49A

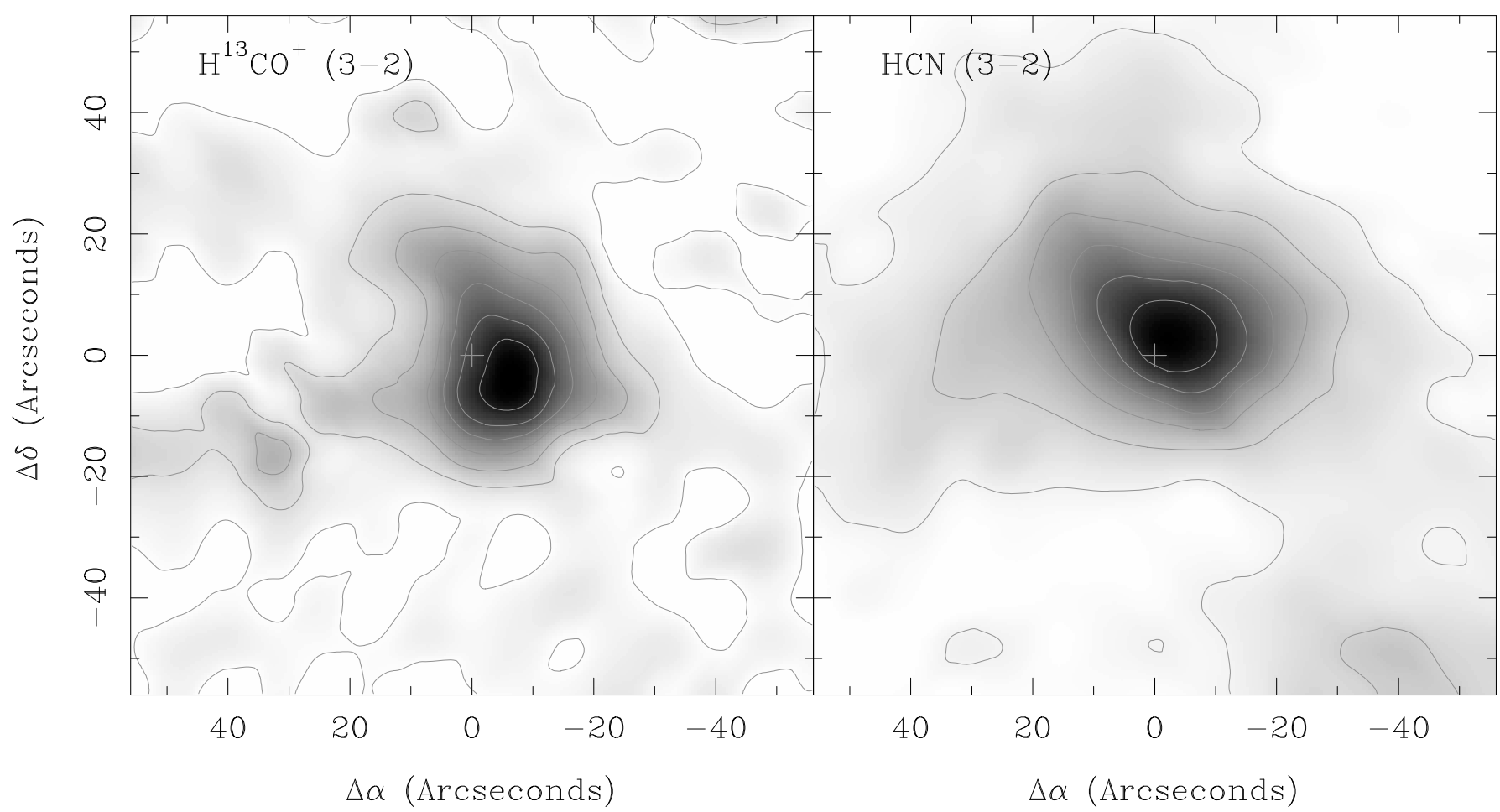

Fig. 3. Maps of the integrated intensity $\left(\int T_{\mathrm{A}}^{*} \mathrm{~d} V\right.$ ) across $\mathrm{W} 49: \mathrm{H}^{13} \mathrm{CO}^{+} 3-2(l e f t)$ and $\mathrm{HCN} 3-2$ (right). The contours show 0 to $100 \%$ of the peak integrated intensity (in steps of $15 \%$ ), which is $24 \mathrm{~K} \mathrm{~km} \mathrm{~s}^{-1}$ for $\mathrm{H}^{13} \mathrm{CO}^{+}$and 172 for $\mathrm{HCN}$.

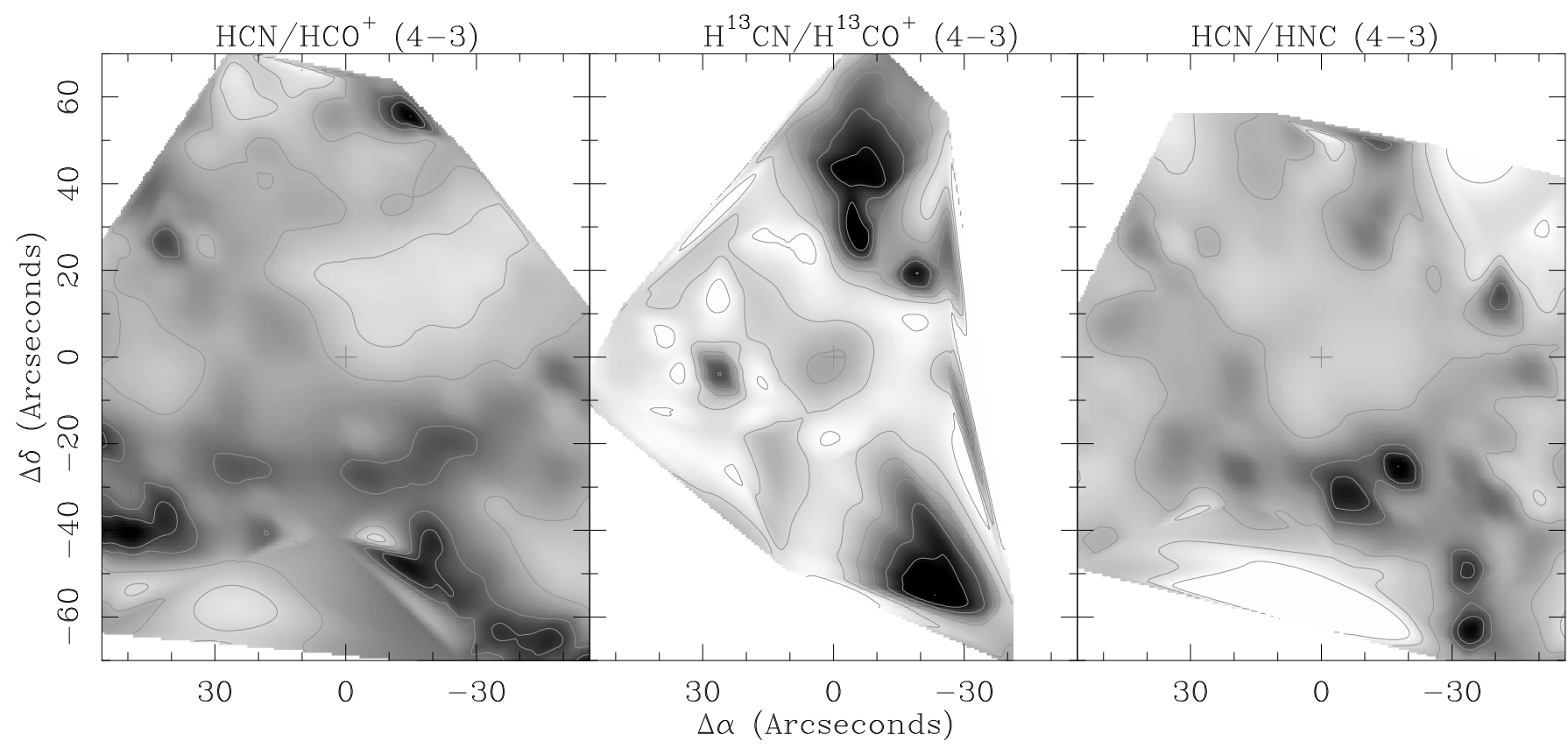

Fig. 4. Maps of the $\mathrm{HCN} / \mathrm{HCO}^{+}, \mathrm{H}^{13} \mathrm{CN} / \mathrm{H}^{13} \mathrm{CO}^{+}$, and $\mathrm{HCN} / \mathrm{HNC} J=4-3$ integrated intensity $\left(\int T_{\mathrm{A}}^{*} \mathrm{~d} V\right)$ ratios across $\mathrm{W} 49 \mathrm{~A}$ (greyscale and contours). Contours show 10 to $100 \%$ of the peak values (in steps of $15 \%$ ) which are 1.4 for $\mathrm{HCN} / \mathrm{HCO}^{+}, 4.9$ for $\mathrm{H}^{13} \mathrm{CN} / \mathrm{H}^{13} \mathrm{CO}^{+}$, and 10.3 for $\mathrm{HCN} / \mathrm{HNC}$. A cutoff integrated intensity was applied to each transition before calculating the ratio to avoid divide-by-zero errors in low signal regions of the maps. The cutoff values are $1.5 \mathrm{~K} \mathrm{~km} \mathrm{~s}^{-1}$ for $\mathrm{HCN}, 0.5 \mathrm{~K} \mathrm{~km} \mathrm{~s}^{-1}$ for $\mathrm{HNC}, 3.0 \mathrm{~K} \mathrm{~km} \mathrm{~s}^{-1}$ for $\mathrm{HCO}^{+}, 0.7 \mathrm{~K} \mathrm{~km} \mathrm{~s}^{-1}$ for $\mathrm{H}^{13} \mathrm{CN}$, and $0.3 \mathrm{~K} \mathrm{~km} \mathrm{~s}^{-1}$ for $\mathrm{H}^{13} \mathrm{CO}^{+}$.

limited dynamic range of the maps in Fig. 4 and the fact that the $\mathrm{HCN} / \mathrm{H}^{13} \mathrm{CN}, \mathrm{HNC} / \mathrm{HN}^{13} \mathrm{C}$ and $\mathrm{HCO}^{+} / \mathrm{H}^{13} \mathrm{CO}^{+}$intensity ratios are 5-10. These ratios are wel below the isotopic abundance ratio of 60 , which indicates line optical depths of $\sim 10$, assuming that the ${ }^{13} \mathrm{C}$-substituted lines are optically thin.

\subsection{Line profiles}

Figure 5 shows the $\mathrm{HCN}$ and $\mathrm{HCO}^{+}$line profiles observed towards $5 \times 5$ pixels $(37.5 \times 37.5$ arcsec $)$ centred at $\mathrm{RA}=19: 10: 13.4 ;$ Dec $=09: 06: 14$, where the molecular 

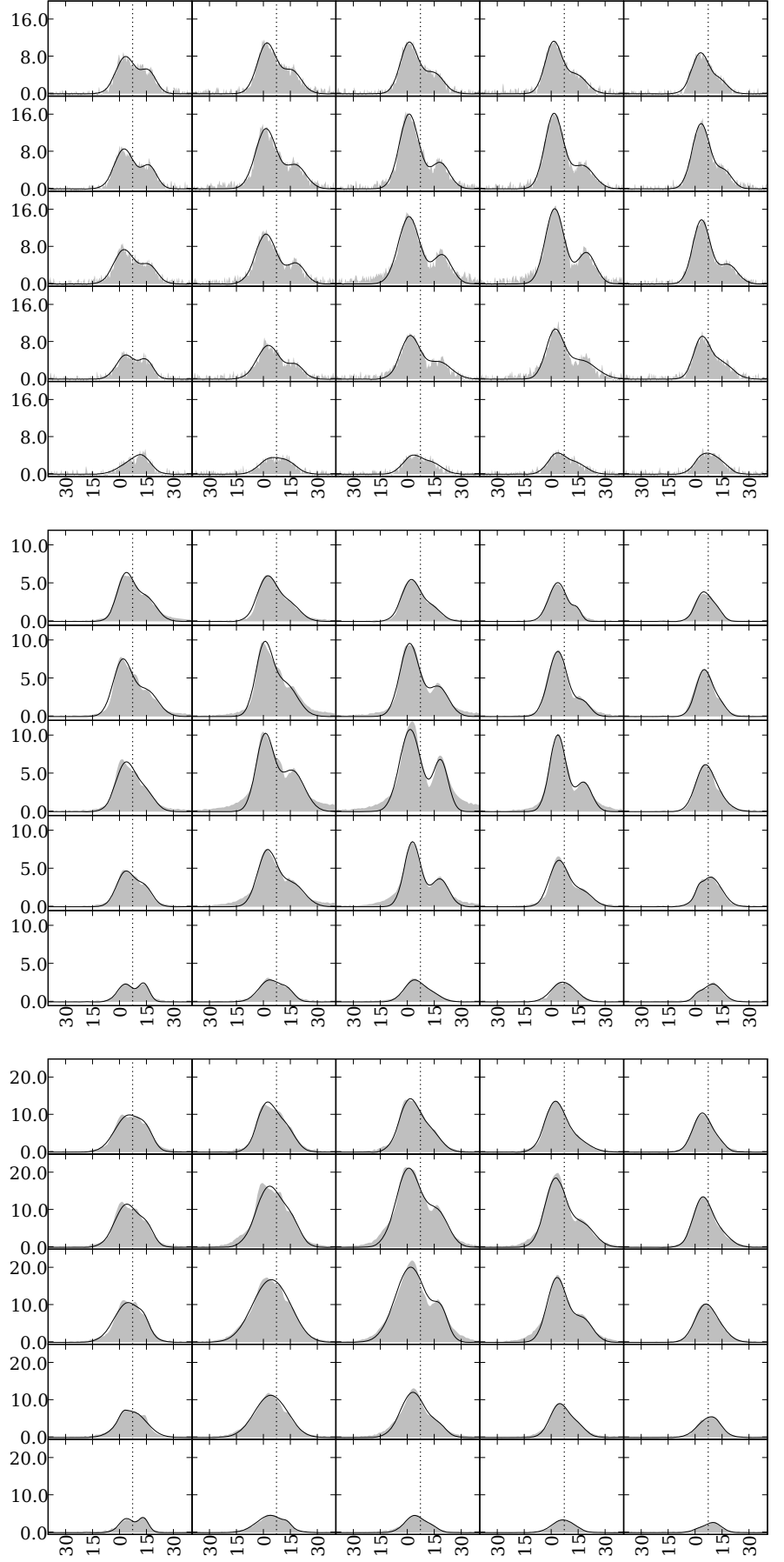

Fig. 5. Observed spectral lines (shaded grey) and Gaussian fit profiles (black lines) towards a $37.5^{\prime \prime} \times 37.5^{\prime \prime}$ grid centred at RA = 19:10:13.4; Dec: 09:06:14. The $X$-axis is velocity relative to the line rest frequency $\left(\mathrm{km} \mathrm{s}^{-1}\right.$ ); the $Y$-axis is $T_{\mathrm{mb}}(\mathrm{K})$. HCN $J=3-2$ (top); HCN $J=4-3$ (middle); $\mathrm{HCO}^{+} J=4-3$ (bottom). The dashed vertical line on each plot is at the expected source velocity of $7 \mathrm{~km} \mathrm{~s}^{-1}$.

emission peaks. Many of the profiles are double peaked and the rest are asymmetric. All profiles are best fitted by two Gaussians; the combined fit profiles are also shown. Figure 6 shows histograms of the number of spectra in each map as a function of the peak velocity of each Gaussian fit. The bin width of $3 \mathrm{~km} \mathrm{~s}^{-1}$ was chosen to be large enough to obtain significant signal in each bin, yet small enough to be able to locate the peaks of the distribution. For all three species, the lower-velocity component
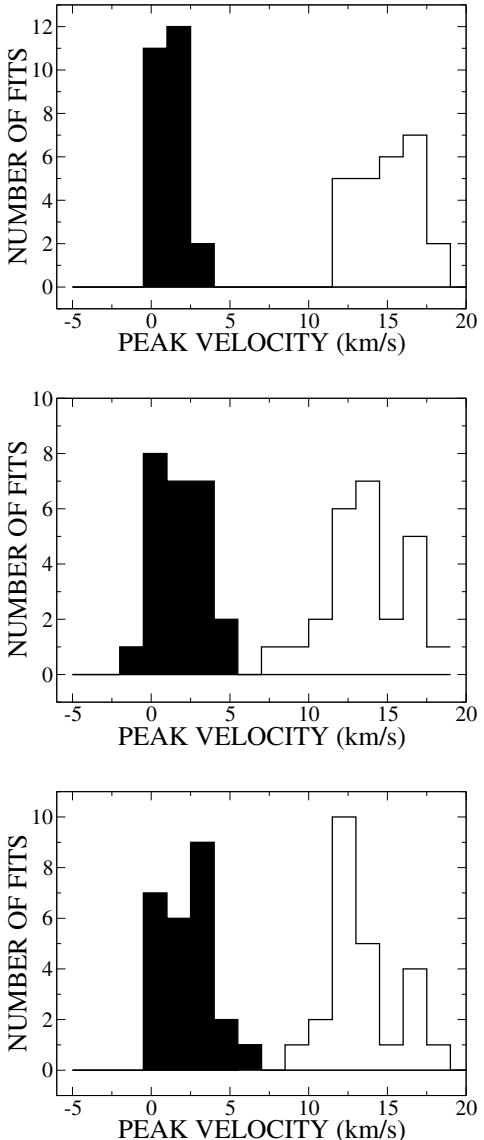

Fig. 6. Histograms of the peak velocity for the spectral lines observed towards the centre of W49A (see Fig. 5) measured with double Gaussian fits to the line profiles at each map position. The number of lower velocity components is black and the number of higher velocity components is white. From top to bottom: $\mathrm{HCN} J=3-2$; $\mathrm{HCN} J=$ $4-3 ; \mathrm{HCO}^{+} J=4-3$.

of each line peaks between 0 and $+5 \mathrm{~km} \mathrm{~s}^{-1}$ while the highervelocity component peaks between +12 and $+15 \mathrm{~km} \mathrm{~s}^{-1}$. On the other hand, our observations of the rarer isotopologues show single-peaked line profiles (Figs. 8 and 9) which peak close to the source velocity of $+7-8 \mathrm{~km} \mathrm{~s}^{-1}$ (Welch et al. 1987; Jaffe et al. 1987). Thus, the $\mathrm{HCN}$ and $\mathrm{HCO}^{+}$lines are self-absorbed and highly optically thick.

In the central region of W49A, the blue-shifted peaks of the main isotopic lines are brighter than the red-shifted peaks by $50-100 \%$, which is a classic sign of infall (e.g. Zhou et al. 1993). Infall motion has been observed toward similar regions: on large scales toward W43 (Motte et al. 2003) and on smaller scales toward W51 (Zhang \& Ho 1997). In the case of W49A, Dickel \& Auer (1994) suggest large scale free-fall collapse of the molecular cloud towards a central HII region based on $\mathrm{HCO}^{+} J=1-0$ and 3-2 line profiles. However, Serabyn et al. (1993) find double-peaked line profiles in both CS and $\mathrm{C}^{34} \mathrm{~S}$ and argue that the starburst in W49A is due to the collision of two clouds. Also, our maps show that although "infall-type" line profiles are seen in the east-west direction, to the north and south there are regions where the red-shifted emission is stronger than the blue, suggesting outflowing motions in this direction (see also Sect. 3.3.4). Finally, the clump of emission in the southwest corner is characterised by single-peaked lines close to the 
expected source velocity. We conclude that the gas kinematics in W49A is complex, with infall and outflow motions probably occurring simultaneously. Imaging of W49A at high spatial and spectral resolution is necessary for a better understanding of its gas kinematics.

In order to investigate the velocity profiles across the maps, Fig. 7 shows integrated intensity maps at the velocities of the $\mathrm{HCN}, \mathrm{HCO}^{+}$, and $\mathrm{HNC} J=4-3$ fit components as well as at the expected source velocity. While the spatial distributions are significantly different at the different velocity ranges, the maps of all molecules over the same velocity range are very similar. The blue-shifted emission is stronger at the east and west edges of the map, while the red-shifted emission is more extended in the north-south direction. The maps in Fig. 7 also suggest the existence of unresolved substructure: possible effects of this substructure will be discussed in Sect. 3.3.

Based on Fig. 7, we select three regions of the map away from the source centre to study in more detail: the "South-West clump" (centred at RA = 19:10:10.6; Dec $=09: 05: 18$ ) is clearly seen at the source velocity and in the red-shifted component, but is much fainter in the blue-shifted component; the "Northern clump" $(\mathrm{RA}=19: 10: 13.6$; Dec $=09: 06: 48)$ is a peak in the integrated intensity seen only in the red-shifted component; while the "Eastern tail" ( $\mathrm{RA}=19: 10: 16.6$; Dec $=09: 05: 48)$ is much stronger at the velocity of the blue-shifted component.

\subsection{Column densities}

To derive the column densities of $\mathrm{HCN}, \mathrm{HNC}$ and $\mathrm{HCO}^{+}$, we use the lines of their isotopically substituted species, which have a lower optical depth than the main species. Figures 8 and 9 show the observed profiles of these lines, along with single Gaussian fits. Although the ${ }^{13} \mathrm{C}$-substituted lines are fitted reasonably well, these lines are broader than the weaker isotopologues and show somewhat asymmetric profiles, so their optical depths may not be negligible. This is shown in more detail in Fig. 10, which compares the line profiles of the $\mathrm{HCO}^{+}, \mathrm{HCN}$, and $\mathrm{HNC}$ (and isotopologue) spectra towards the central pixel of the W49A map. The ${ }^{13} \mathrm{C}$-substituted lines do peak at $\sim 8 \mathrm{~km} \mathrm{~s}^{-1}$, close to the source velocity, but, as well as being asymmetric, the $\mathrm{H}^{13} \mathrm{CN} J=4-3$ line $F W H M$ is $\sim 1.4$ times that of the $\mathrm{HC}^{15} \mathrm{~N} J=4-3$ line. For this reason we have, wherever possible, based our calculations of the $\mathrm{HCO}^{+}$and $\mathrm{HCN}$ column densities and the DCN/HCN ratios on the $\mathrm{HC}^{15} \mathrm{~N}$ and $\mathrm{HC}^{18} \mathrm{O}^{+}$lines.

We use the RADEX program (Van der Tak et al. 2007) to estimate column densities from the observed widths and peak intensities of the Gaussian fits to the line emission. This program performs statistical equilibrium calculations including collisional and radiative processes, and treats optical depth effects with an escape probability formalism. The program assumes that the optical depth is independent of velocity, which is appropriate since we are modeling the velocity-integrated line intensities. Molecular input data for $\mathrm{HCN}$ and $\mathrm{HCO}^{+}$are taken from the LAMDA database (Schöier et al. 2005); we assume that collisional rates do not change upon isotopic substitution which is probably valid given the identical electronic structure. We adopt the HCN collision rates for HNC, which assumption is a potential source of error since the two species have somewhat different dipole moments (3.0 vs. 3.3 D). Very recently, Sarrasin et al. (2010) and Dumouchel et al. (2010) have presented collision data for $\mathrm{HCN}$ and $\mathrm{HNC}$ with $\mathrm{He}$; the impact of these data on the present work will be analyzed in a forthcoming paper.
Since the emission is spatially extended, we assume a beam filling factor of unity for all lines. Due to the presence of substructure within the JCMT beam (e.g., Dickel et al. 1999), this assumption provides lower limits on the line optical depths and molecular column densities. The measured line widths provide an estimate of these limits. For example, the width of the $\mathrm{H}^{13} \mathrm{CN} 4-3$ line toward the source centre, which is $1.4 \times$ larger than that of the $\mathrm{HC}^{15} \mathrm{~N}$ line, suggests a $3-5 \times$ higher optical depth than the value of $\tau=0.2$ estimated with RADEX. However, since much of our analysis is based on line ratios, the optical depths of individual lines are not a major concern. Section 4 provides further discussion on the effect of unresolved substructure.

For $\mathrm{HCN}$ and $\mathrm{HCO}^{+}$, the intensities of two transitions are known, which allows an estimate of the ambient physical conditions (kinetic temperature and $\mathrm{H}_{2}$ volume density). In the case of $\mathrm{HNC}$, only one transition was observed, and we have adopted the temperature and density found for $\mathrm{HCN}$ and $\mathrm{HCO}^{+}$.

\subsubsection{The source centre}

We calculate the column density of the central source adopting $T_{\text {kin }}=100 \mathrm{~K}$ and $n\left(\mathrm{H}_{2}\right)=2 \times 10^{6} \mathrm{~cm}^{-3}$ (Tables 3 and 4). Using these physical conditions, $T_{\mathrm{ex}}\left(\mathrm{H}^{13} \mathrm{CO}^{+} J=3-2\right) \sim T_{\text {kin }}$, and the $\mathrm{HCO}^{+}$column density determinations from $\mathrm{H}^{13} \mathrm{CO}^{+} J=3-2$, $\mathrm{H}^{13} \mathrm{CO}^{+} J=4-3$, and $\mathrm{HC}^{18} \mathrm{O}^{+} J=4-3$ towards the source centre all agree to within a factor of 2 , with the column density determined from the $J=4-3$ and $J=3-2$ transitions becoming more discrepant at lower density. Over the range $T_{\text {kin }}=75-200 \mathrm{~K}$ and $n\left(\mathrm{H}_{2}\right)=5 \times 10^{5}-5 \times 10^{6} \mathrm{~cm}^{-3}$ (Sect. 1), the derived column density determinations from the $\mathrm{H}^{13} \mathrm{CN} J=4-3$ transitions agree very well with those from the $\mathrm{HC}^{15} \mathrm{~N} J=4-3$ transitions, assuming isotopic ratios appropriate for the local ISM (Wilson \& Rood 1994). The calculated HCN and DCN column densities fall by a factor of 2 as the kinetic temperature increases from 75 to $200 \mathrm{~K}$ or by almost an order of magnitude if the density increases from $5 \times 10^{5} \mathrm{~cm}^{-3}$ to $5 \times 10^{6} \mathrm{~cm}^{-3}$. Over this range of temperature and density, the calculated excitation temperatures for the HCN and DCN transitions are similar to each other and increase from $\sim 15 \mathrm{~K}$ for $T_{\text {kin }}=75 \mathrm{~K}$ and $n\left(\mathrm{H}_{2}\right)=5 \times 10^{5} \mathrm{~cm}^{-3}$ to almost $40 \mathrm{~K}$ for $T_{\text {kin }}=200 \mathrm{~K}$ and $n\left(\mathrm{H}_{2}\right)=5 \times 10^{6} \mathrm{~cm}^{-3}$.

Tables 3 and 4 list fit parameters and resulting column densities, optical depths and excitation temperatures calculated by RADEX for the central pixels. As we expect, optical depths are generally higher for the transitions of the ${ }^{13} \mathrm{C}$-substituted molecules than for those containing rarer isotopes. The excitation temperatures are $\sim 40 \mathrm{~K}$ for the $\mathrm{H}^{13} \mathrm{CO}^{+}$and $\mathrm{HC}^{18} \mathrm{O}^{+} \mathrm{J}=$ 4-3 lines $\left(\sim 86 \mathrm{~K}\right.$ for the $\mathrm{H}^{13} \mathrm{CO}^{+} \mathrm{J}=3-2$ lines), $\sim 16 \mathrm{~K}$ for the $\mathrm{H}^{13} \mathrm{CN}$ and $\mathrm{HC}^{15} \mathrm{~N} J=4-3$ lines, and $\sim 18 \mathrm{~K}$ for the DCN $J=5-4$ lines. We estimate $N\left(\mathrm{HCO}^{+}\right) \sim 10^{14}-10^{15} \mathrm{~cm}^{-2}$, $N(\mathrm{HCN}) \sim$ a few times $10^{15} \mathrm{~cm}^{-2}, \mathrm{DCN} / \mathrm{HCN}=4 \times 10^{-4}-3 \times$ $10^{-3}$, and $\mathrm{HCN} / \mathrm{HCO}^{+}=3-10$. Based on $3 \sigma$ rms noise levels in the $\mathrm{DCO}^{+} J=5-4$ spectra, we calculate upper limits on the $\mathrm{DCO}^{+} / \mathrm{HCO}^{+}$ratios towards the centre of $\mathrm{W} 49 \mathrm{~A}$ to be $3-4 \times 10^{-4}$ for $T_{\text {ex }}=20-40 \mathrm{~K}$.

We have also estimated the HNC column density towards the centre of W49A, from the $J=4-3$ transition. For $T_{\text {kin }}=100 \mathrm{~K}$ and $n\left(\mathrm{H}_{2}\right)=2 \times 10^{6} \mathrm{~cm}^{-3}$, we find $N(\mathrm{HNC})=10^{14} \mathrm{~cm}^{-2}$ so the $\mathrm{HCN} / \mathrm{HNC}$ ratio is $\sim 50$. The result that $N(\mathrm{HCN}) / N\left(\mathrm{HCO}^{+}\right)$ratios are $>1$ is contrary to that found by naively taking line ratios of the main $J=4-3$ lines (Fig. 4; Table 2), which shows the importance of taking excitation effects into account when estimating column densities or abundance ratios. 


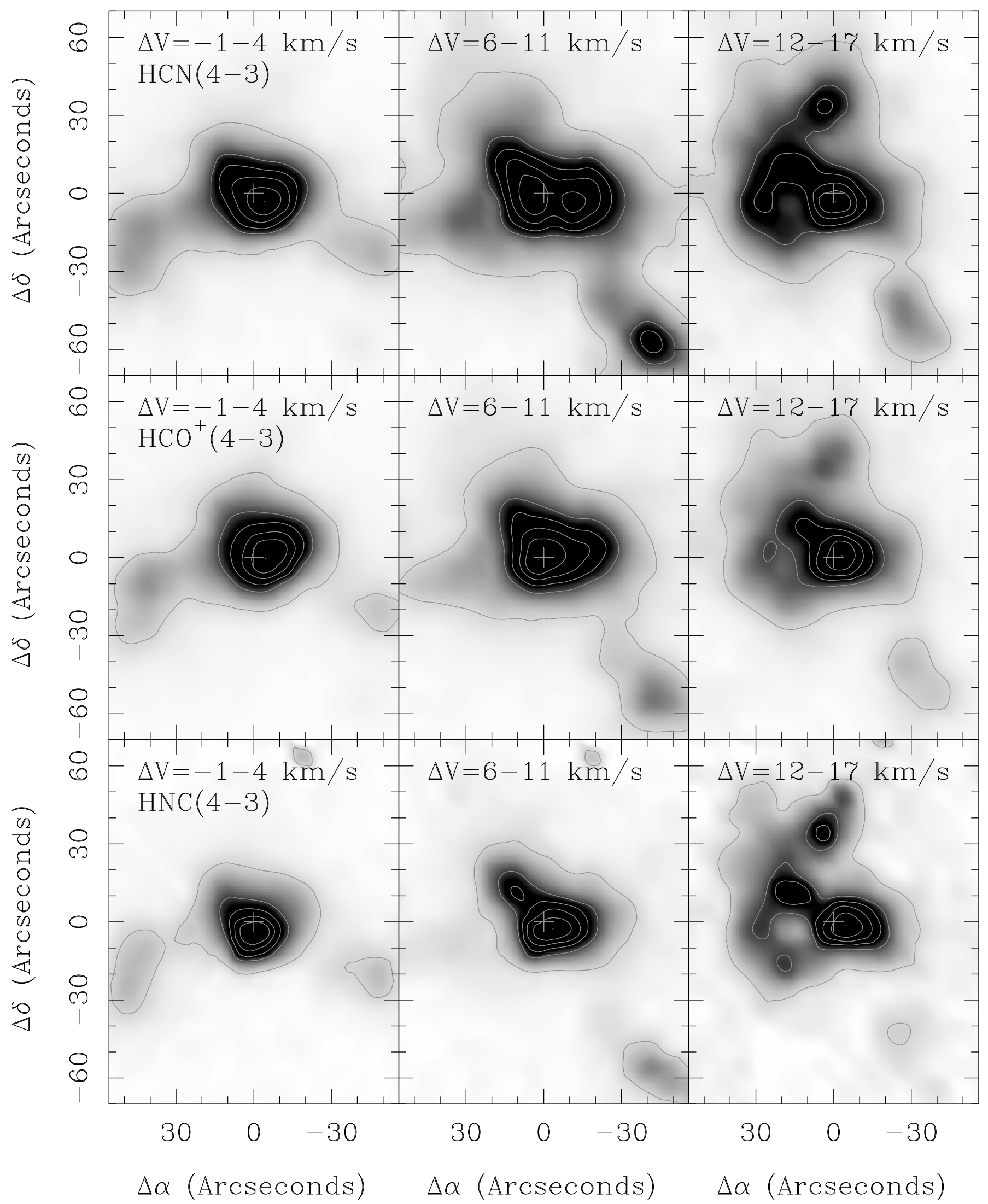

Fig. 7. Maps of the integrated intensity $\left(\int T_{\mathrm{A}}^{*} \mathrm{~d} V\right)$ across W49A over $5 \mathrm{~km} \mathrm{~s}^{-1}$ velocity ranges. Left column: -1 to $+4 \mathrm{~km} \mathrm{~s}^{-1}$ (the blue-shifted peak); middle column: +6 to $+11 \mathrm{~km} \mathrm{~s}^{-1}$ (the expected source velocity); right column: +12 to $+17 \mathrm{~km} \mathrm{~s}^{-1}$ (the red-shifted peak). Top row: HCN $J=4-3$; centre row: $\mathrm{HCO}^{+} J=4-3$; bottom row: HNC $J=4-3$. The contours show 10 to $100 \%$ of the peak integrated intensity in steps of $15 \%$. The peak intensities are 33,18, and $16 \mathrm{~K} \mathrm{~km} \mathrm{~s}^{-1}$, for the blue-shifted, central velocity, and red-shifted HCN $J=4-3$ emission, respectively; 63, 43, and $33 \mathrm{~K} \mathrm{~km} \mathrm{~s}^{-1}$ for $\mathrm{HCO}^{+}$; and 16,12 , and $5 \mathrm{~K} \mathrm{~km} \mathrm{~s}^{-1}$ for $\mathrm{HNC}$. 

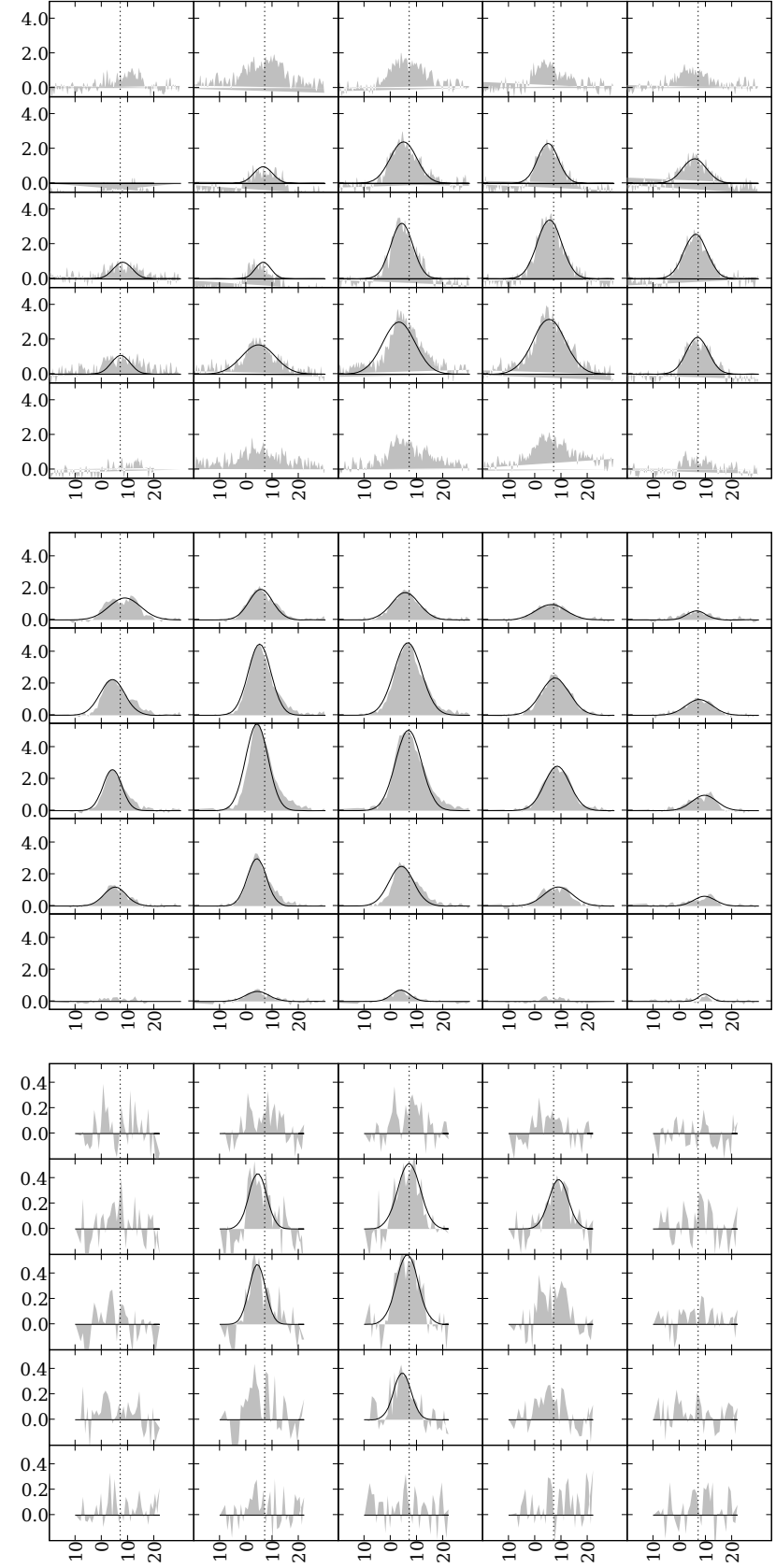

Fig. 8. As Fig. 5 but, top to bottom: $\mathrm{H}^{13} \mathrm{CO}^{+} J=3-2 ; \mathrm{H}^{13} \mathrm{CO}^{+} J=4-3$; $\mathrm{HC}^{18} \mathrm{O}^{+} J=4-3$.

\subsubsection{The South-West clump}

As Figs. 8 and 9 show, the strength of the isotope-substituted transitions falls rapidly away from the source centre. Only the ${ }^{13} \mathrm{C}$-substituted $J=4-3$ lines are observed towards the SW-clump. Figure 11 shows the detected lines along with Gaussian fit profiles. The lines peak close to the expected source velocity and are well-fitted by single Gaussian profiles. Again, we have estimated column densities using RADEX, this time varying the physical parameters over the ranges $T_{\text {kin }}=20-100 \mathrm{~K}$ and $n\left(\mathrm{H}_{2}\right)=5 \times 10^{3}-10^{6} \mathrm{~cm}^{-3}$. We find that, unless $n\left(\mathrm{H}_{2}\right)>$ $10^{5} \mathrm{~cm}^{-3}$, the $\mathrm{HCN}$ and $\mathrm{HCO}^{+}$column densities towards the clump are at least 10 times higher than towards the source centre, due to the high critical densities of these transitions. As this would imply fractional abundances at least 2 orders of
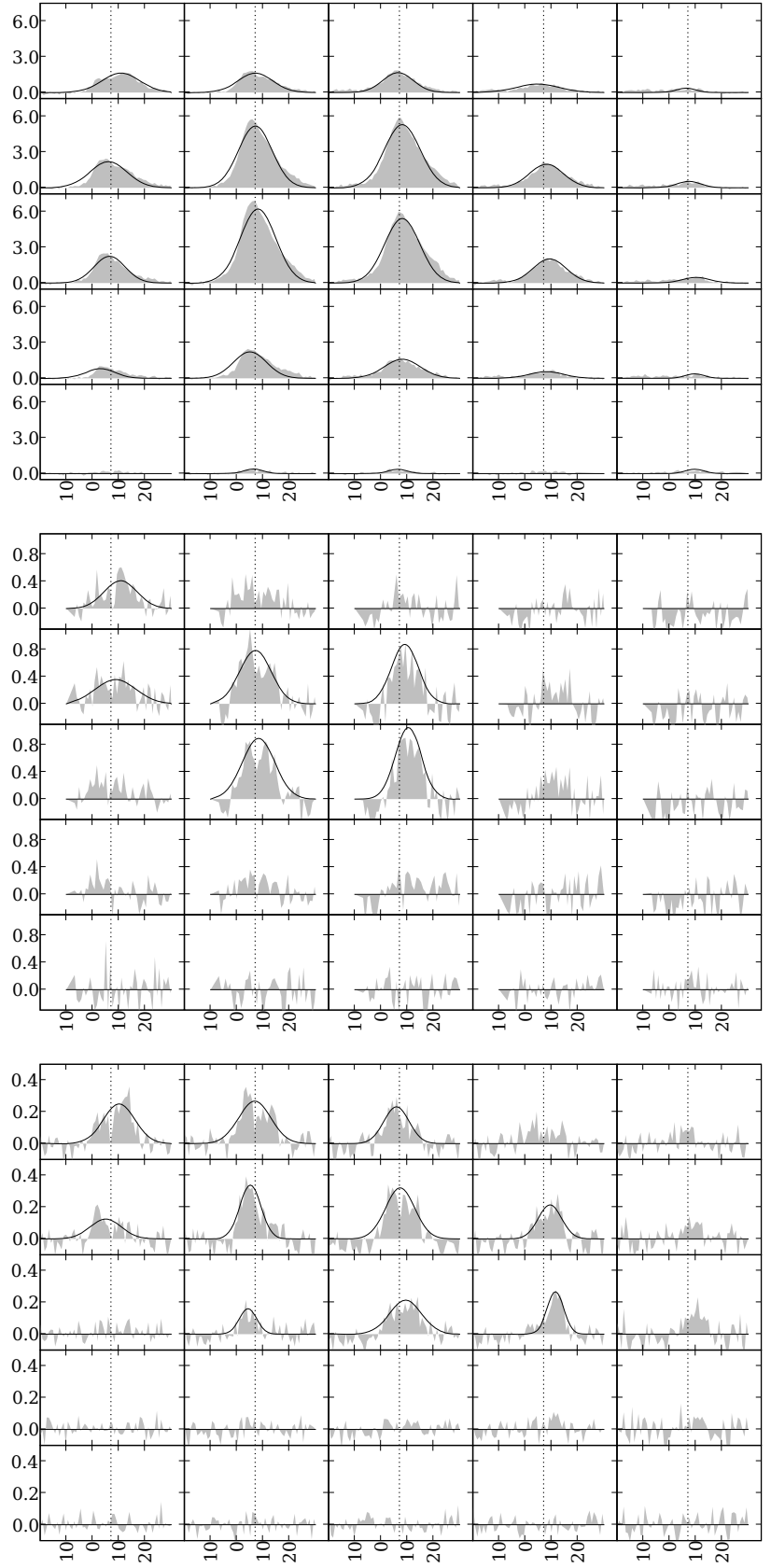

Fig. 9. As Fig. 5 but, top to bottom: $\mathrm{H}^{13} \mathrm{CN} J=4-3$; $\mathrm{HC}^{15} \mathrm{~N} J=4-3$; $\mathrm{DCN}^{+} J=5-4$.

magnitude higher at the clump position, it seems more likely that this gas also has a relatively high density of $10^{5}-10^{6} \mathrm{~cm}^{-3}$.

We have tried to constrain the kinetic temperature by looking at the upper limits on the $\mathrm{DCN} / \mathrm{HCN}$ and $\mathrm{DCO}^{+} / \mathrm{HCO}^{+}$ratios and at the $\mathrm{HCN} / \mathrm{HNC}$ ratio. The upper level energies of the $\mathrm{DCN}$ and $\mathrm{DCO}^{+} 5-4$ transitions are $\sim 50 \mathrm{~K}$, though, so we would not expect these lines to be very strong at lower temperatures. For $T_{\text {kin }}=20 \mathrm{~K}$, the upper limit on the DCN/HCN ratio from the observations is 0.01 whereas steady-state chemical models predict $\mathrm{DCN} / \mathrm{HCN} \sim 0.02$ at $20 \mathrm{~K}$ (see Sect. 4.2), slight evidence that the kinetic temperature in the SW clump is higher than $20 \mathrm{~K}$. The HCN and HNC column densities predicted by RADEX give a $\mathrm{HCN} / \mathrm{HNC}$ ratio $\sim 7$, which suggests that this region has a lower temperature than the source centre. 
Table 3. Estimated $\mathrm{HCO}^{+}$column densities towards the source centre.

\begin{tabular}{llccccccc}
\hline \hline $\begin{array}{l}\text { Offset } \\
\left({ }^{\prime \prime},{ }^{\prime}\right)\end{array}$ & Transition & $\begin{array}{l}\text { Velocity } \\
\left(\mathrm{km} \mathrm{s}^{-1}\right)\end{array}$ & $\begin{array}{c}T_{\mathrm{mb}} \\
(\mathrm{K})\end{array}$ & $\begin{array}{c}F W H M \\
\left(\mathrm{~km} \mathrm{~s}^{-1}\right)\end{array}$ & $\begin{array}{c}T_{\mathrm{ex}} \\
(\mathrm{K})\end{array}$ & $\tau$ & $\begin{array}{c}N \\
\left(10^{12} \mathrm{~cm}^{-2}\right)\end{array}$ & $\begin{array}{c}N\left(\mathrm{HCO}^{+}\right)^{b} \\
\left(10^{14} \mathrm{~cm}^{-2}\right)\end{array}$ \\
\hline $15.0,-7.5$ & $\mathrm{H}^{13} \mathrm{CO}^{+} J=4-3$ & 5.0 & 1.2 & 10.0 & 37.6 & 0.04 & 4.5 & 3.5 \\
$15.0,0.0$ & $\mathrm{H}^{13} \mathrm{CO}^{+} J=4-3$ & 4.0 & 2.6 & 8.4 & 38.3 & 0.08 & 8.3 & 6.4 \\
$15.0,7.5$ & $\mathrm{H}^{13} \mathrm{CO}^{+} J=4-3$ & 4.0 & 2.2 & 11.1 & 38.1 & 0.07 & 9.5 & 7.3 \\
$15.0,15.0$ & $\mathrm{H}^{13} \mathrm{CO}^{+} J=4-3$ & 8.8 & 1.4 & 14.2 & 37.7 & 0.04 & 7.3 & 5.6 \\
$7.5,-15.0$ & $\mathrm{H}^{13} \mathrm{CO}^{+} J=4-3$ & 4.2 & 0.6 & 10.4 & 37.3 & 0.02 & 2.4 & 1.9 \\
$7.5,-7.5$ & $\mathrm{H}^{13} \mathrm{CO}^{+} J=4-3$ & 4.0 & 3.0 & 8.7 & 38.5 & 0.10 & 9.9 & 7.7 \\
$7.5,0.0$ & $\mathrm{HC}^{18} \mathrm{O}^{+} J=4-3$ & 4.2 & 0.5 & 7.4 & 38.6 & 0.01 & 1.3 & 7.2 \\
$7.5,7.5$ & $\mathrm{HC}^{18} \mathrm{O}^{+} J=4-3$ & 4.3 & 0.4 & 8.1 & 38.6 & 0.01 & 1.3 & 7.3 \\
$7.5,15.0$ & $\mathrm{H}^{13} \mathrm{CO}^{+} J=4-3$ & 5.5 & 1.9 & 10.6 & 38.0 & 0.06 & 7.7 & 6.0 \\
$0.0,-15.0$ & $\mathrm{H}^{13} \mathrm{CO}^{+} J=4-3$ & 3.8 & 0.7 & 8.2 & 37.3 & 0.02 & 2.1 & 1.7 \\
$0.0,-7.5$ & $\mathrm{HC}^{18} \mathrm{O}^{+} J=4-3$ & 4.4 & 0.4 & 7.8 & 38.6 & 0.01 & 1.1 & 6.0 \\
$0.0,0.0$ & $\mathrm{HC}^{18} \mathrm{O}^{+} J=4-3$ & 6.2 & 0.5 & 9.7 & 38.7 & 0.02 & 2.0 & 11. \\
$0.0,7.5$ & $\mathrm{HC}^{18} \mathrm{O}^{+} J=4-3$ & 6.9 & 0.5 & 10.2 & 38.6 & 0.02 & 1.9 & 11. \\
$0.0,15.0$ & $\mathrm{H}^{13} \mathrm{CO}^{+} J=4-3$ & 5.5 & 1.7 & 12.3 & 37.9 & 0.06 & 8.0 & 6.2 \\
$-7.5,-15.0$ & $\mathrm{H}^{13} \mathrm{CO}^{+} J=3-2$ & 6.4 & 1.7 & 27.3 & 86.2 & 0.02 & 20. & 16. \\
$-7.5,-7.5$ & $\mathrm{H}^{13} \mathrm{CO}^{+} J=4-3$ & 8.8 & 1.2 & 12.7 & 37.6 & 0.04 & 5.7 & 4.4 \\
$-7.5,0.0$ & $\mathrm{H}^{13} \mathrm{CO}^{+} J=4-3$ & 8.3 & 2.8 & 11.5 & 38.4 & 0.09 & 12. & 9.4 \\
$-7.5,7.5$ & $\mathrm{HC}^{18} \mathrm{O}^{+} J=4-3$ & 8.9 & 0.4 & 8.6 & 38.6 & 0.01 & 1.2 & 7.0 \\
$-7.5,15.0$ & $\mathrm{H}^{13} \mathrm{CO}^{+} J=4-3$ & 6.1 & 1.0 & 13.3 & 37.5 & 0.03 & 4.9 & 3.8 \\
$-15.0,-15.0$ & $\mathrm{H}^{13} \mathrm{CO}^{+} J=4-3$ & 9.6 & 0.5 & 5.6 & 37.2 & 0.02 & 1.0 & 0.8 \\
$-15.0,-7.5$ & $\mathrm{H}^{13} \mathrm{CO}^{+} J=4-3$ & 9.4 & 0.6 & 9.6 & 37.3 & 0.02 & 2.2 & 1.7 \\
$-15.0,0.0$ & $\mathrm{H}^{13} \mathrm{CO}^{+} J=4-3$ & 9.4 & 1.0 & 11.1 & 37.5 & 0.03 & 4.0 & 3.1 \\
$-15.0,7.5$ & $\mathrm{H}^{13} \mathrm{CO}^{+} J=4-3$ & 7.4 & 1.0 & 12.0 & 37.5 & 0.03 & 4.4 & 3.4 \\
$-15.0,15.0$ & $\mathrm{H}^{13} \mathrm{CO}^{+} J=4-3$ & 6.4 & 0.6 & 8.4 & 37.3 & 0.02 & 1.8 & 1.4 \\
\hline
\end{tabular}

Notes. $\mathrm{HCO}^{+}$column densities are calculated with RADEX assuming $T_{\text {kin }}=100 \mathrm{~K}$ and $n\left(\mathrm{H}_{2}\right)=2 \times 10^{6} \mathrm{~cm}^{-3}$. For the offset positions where $\mathrm{HC}^{18} \mathrm{O}^{+}$was not detected, the data for $\mathrm{H}^{13} \mathrm{CO}^{+} J=4-3$ were used, except for $\left(-7.5^{\prime \prime},-15.0^{\prime \prime}\right)$, where $\mathrm{H}^{13} \mathrm{CO}^{+} J=4-3$ was not detected and $\mathrm{H}^{13} \mathrm{CO}^{+} J=3-2$ was used. For the other positions, where all transitions were detected, data for the $\mathrm{HC}^{18} \mathrm{O}^{+} J=4-3$ transition are listed and we note that the column density determinations from the $\mathrm{H}^{13} \mathrm{CO}^{+} J=4-3$ line agree with these to within $\pm 50 \%$.

(a) Offsets from RA = 19:10:13.4, Dec $=$ 09:06:14; ${ }^{(b)}$ based on ${ }^{12} \mathrm{C} /{ }^{13} \mathrm{C}=77 ;{ }^{16} \mathrm{O} /{ }^{18} \mathrm{O}=560$ (Wilson \& Rood 1994).

Table 4. Estimated HCN and DCN column densities towards the source centre.

\begin{tabular}{|c|c|c|c|c|c|c|c|c|c|c|}
\hline $\begin{array}{l}\text { Offset }^{a} \\
\left({ }^{\prime \prime},{ }^{\prime}\right)\end{array}$ & Transition & $\begin{array}{l}\text { Velocity } \\
\left(\mathrm{km} \mathrm{s}^{-1}\right)\end{array}$ & $\begin{array}{l}T_{\mathrm{mb}} \\
(\mathrm{K}) \\
\end{array}$ & $\begin{array}{c}F W H M \\
\left(\mathrm{~km} \mathrm{~s}^{-1}\right)\end{array}$ & $\begin{array}{l}T_{\mathrm{ex}} \\
(\mathrm{K})\end{array}$ & $\tau$ & $\begin{array}{c}N \\
\left(10^{12} \mathrm{~cm}^{-2}\right)\end{array}$ & $\begin{array}{c}N(\mathrm{HCN})^{b} \\
\left(10^{15} \mathrm{~cm}^{-2}\right)\end{array}$ & $\begin{array}{l}\frac{N(\mathrm{DCN})}{N(\mathrm{HCN})} \\
\left(\times 10^{3}\right)\end{array}$ & $\frac{N\left(\mathrm{HCN}^{+}\right)}{N\left(\mathrm{HCO}^{+}\right)}$ \\
\hline $15.0,7.5$ & $\mathrm{HC}^{15} \mathrm{~N} J=4-3$ & 8.8 & 0.4 & 18.1 & 16.3 & 0.04 & 7.8 & 3.5 & & 4.8 \\
\hline $15.0,15.0$ & $\mathrm{HC}^{15} \mathrm{~N} J=4-3$ & 10.7 & 0.4 & 14.7 & 16.3 & 0.04 & 7.3 & 3.3 & & 5.8 \\
\hline $7.5,0.0$ & $\mathrm{HC}^{15} \mathrm{~N} J=4-3$ & 8.3 & 0.9 & 14.6 & 16.4 & 0.09 & 16.0 & 7.2 & & 10. \\
\hline $7.5,7.5$ & $\mathrm{HC}^{15} \mathrm{~N} J=4-3$ & 7.1 & 0.8 & 14.1 & 16.4 & 0.08 & 13.6 & 6.1 & & 8.4 \\
\hline $7.5,15.0$ & $\mathrm{H}^{13} \mathrm{CN} J=4-3$ & 7.1 & 1.6 & 15.6 & 16.5 & 0.17 & 31.8 & 2.4 & & 4.1 \\
\hline $0.0,0.0$ & $\mathrm{HC}^{15} \mathrm{~N} J=4-3$ & 10.4 & 1.1 & 11.7 & 16.4 & 0.11 & 15.1 & 6.8 & & 6.2 \\
\hline $0.0,7.5$ & $\mathrm{HC}^{15} \mathrm{~N} J=4-3$ & 9.1 & 0.9 & 12.2 & 16.4 & 0.09 & 13.0 & 5.9 & & 5.4 \\
\hline $0.0,15.0$ & $\mathrm{H}^{13} \mathrm{CN} J=4-3$ & 6.5 & 1.7 & 13.7 & 16.5 & 0.18 & 28.7 & 2.2 & & 3.6 \\
\hline$-7.5,0.0$ & $\mathrm{H}^{13} \mathrm{CN} J=4-3$ & 9.3 & 2.0 & 15.0 & 16.6 & 0.22 & 38.4 & 3.0 & & 3.1 \\
\hline$-7.5,7.5$ & $\mathrm{H}^{13} \mathrm{CN} J=4-3$ & 8.1 & 2.0 & 15.1 & 16.6 & 0.21 & 37.8 & 2.9 & & 4.2 \\
\hline $15.0,7.5$ & $\mathrm{DCN} J=5-4$ & 5.0 & 0.1 & 14.0 & 18.3 & 0.01 & 4.5 & & 1.3 & \\
\hline $15.0,15.0$ & $\mathrm{DCN} J=5-4$ & 10.1 & 0.2 & 14.2 & 18.3 & 0.02 & 9.1 & & 2.8 & \\
\hline $7.5,0.0$ & $\mathrm{DCN} J=5-4$ & 4.2 & 0.2 & 7.8 & 18.3 & 0.02 & 3.2 & & 0.4 & \\
\hline $7.5,7.5$ & $\mathrm{DCN} J=5-4$ & 5.2 & 0.3 & 9.5 & 18.3 & 0.03 & 8.3 & & 1.4 & \\
\hline $7.5,15.0$ & $\mathrm{DCN} J=5-4$ & 6.8 & 0.3 & 14.5 & 18.3 & 0.03 & 10.0 & & 4.1 & \\
\hline $0.0,0.0$ & $\mathrm{DCN} J=5-4$ & 9.3 & 0.2 & 14.4 & 18.3 & 0.02 & 7.9 & & 1.2 & \\
\hline $0.0,7.5$ & $\mathrm{DCN} J=5-4$ & 7.2 & 0.3 & 13.1 & 18.3 & 0.03 & 10.8 & & 1.9 & \\
\hline $0.0,15.0$ & $\mathrm{DCN} J=5-4$ & 5.8 & 0.2 & 11.3 & 18.3 & 0.02 & 6.8 & & 3.1 & \\
\hline$-7.5,0.0$ & $\mathrm{DCN} J=5-4$ & 11.5 & 0.3 & 7.5 & 18.3 & 0.03 & 5.2 & & 1.8 & \\
\hline$-7.5,7.5$ & $\mathrm{DCN} J=5-4$ & 9.4 & 0.2 & 10.4 & 18.3 & 0.02 & 5.8 & & 2.0 & \\
\hline
\end{tabular}

Notes. DCN and HCN column densities are calculated with RADEX assuming $T_{\text {kin }}=100 \mathrm{~K}$ and $n\left(\mathrm{H}_{2}\right)=2 \times 10^{6} \mathrm{~cm}^{-3}$. For the offset positions where $\mathrm{HC}^{15} \mathrm{~N}$ was not detected, the data for $\mathrm{H}^{13} \mathrm{CN}$ are shown; for the other positions, where both were detected, data for the $\mathrm{HC} \mathrm{C}^{15} \mathrm{~N} J=$ 4-3 transition are listed and we note that the column density determinations from the $\mathrm{H}^{13} \mathrm{CN} J=4-3$ line agree with these to within $\pm 35 \%$.

(a) Offsets from RA = 19:10:13.4, Dec = 09:06:14; ${ }^{(b)}$ based on ${ }^{12} \mathrm{C} /{ }^{13} \mathrm{C}=77 ;{ }^{14} \mathrm{~N} /{ }^{15} \mathrm{~N}=450$ (Wilson \& Rood 1994). 

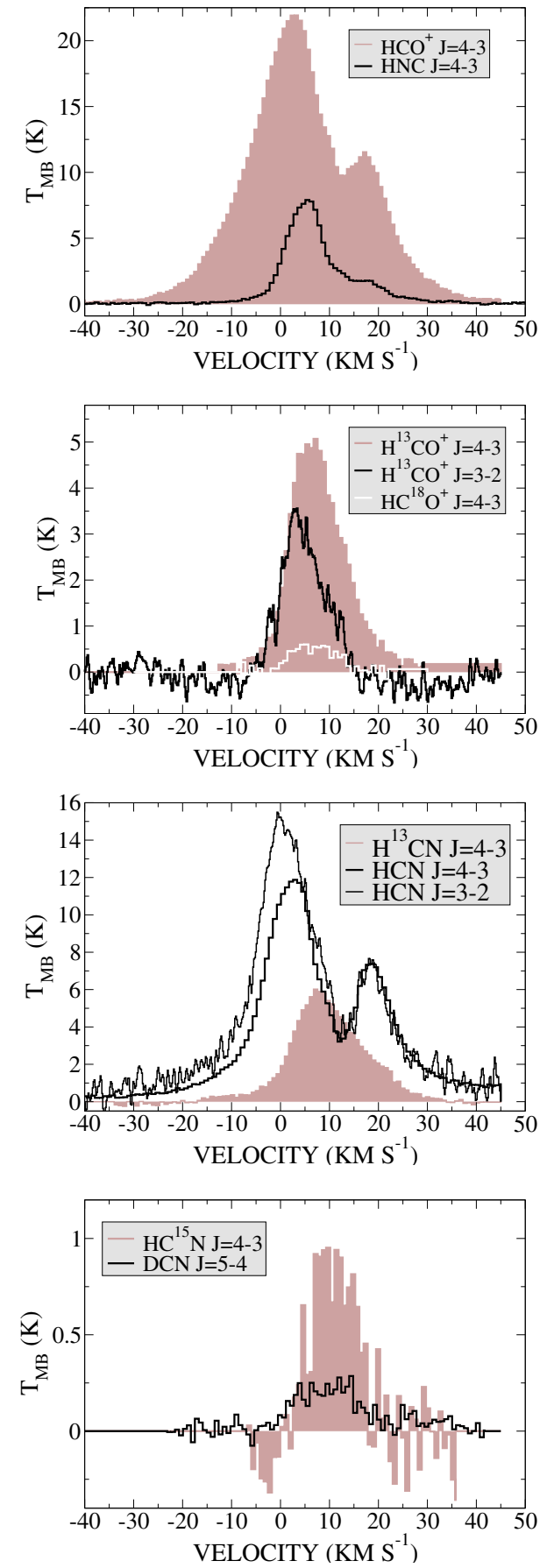

Fig. 10. Line profiles towards the central position ( $\mathrm{RA}=19: 10: 13.4$; Dec $=9: 06: 14)$. Note the difference in beam size between the 4-3 and 3-2 transitions.

Table 5, therefore, lists the line parameters and calculated column densities assuming $T_{\text {kin }}=40 \mathrm{~K}$ and $n\left(\mathrm{H}_{2}\right)=5 \times$ $10^{5} \mathrm{~cm}^{-3}$. This temperature is consistent with that determined by Jaffe et al. (1987) for the outer parts of the cloud (see Sect. 1), although the density is ten times higher than estimated by Welch et al. (1987). We note that the $\mathrm{HCN}$ and $\mathrm{HCO}^{+}$column densities based on the ${ }^{13} \mathrm{C}$ substituted isotopologues, assuming ${ }^{12} \mathrm{C} /{ }^{13} \mathrm{C}=77$, are $4-6$ times higher than those based on observations of the main lines. The upper limits on the column densities of the deuterated species do not put very strong constraints on the deuterium fractionation: $\mathrm{DCN} / \mathrm{HCN}<0.01$
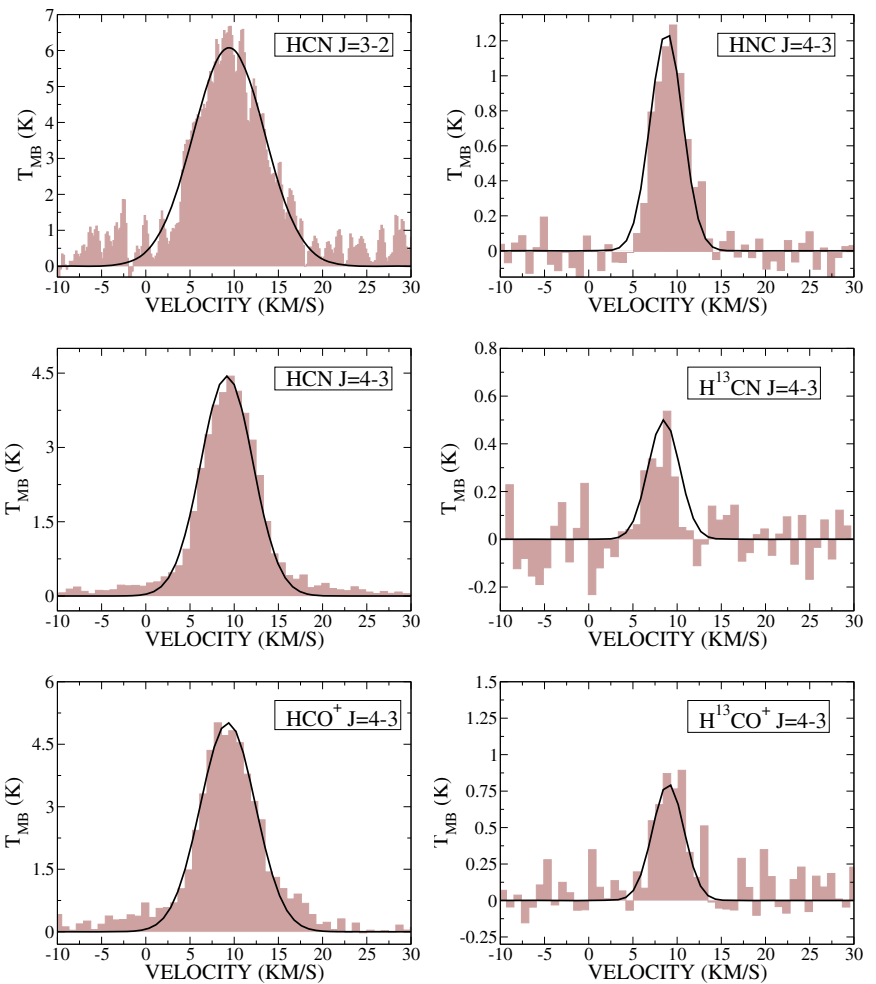

Fig. 11. Observed line profiles (shaded grey) and Gaussian fits (black lines) for the transitions observed towards the "south-west clump" position: $\mathrm{RA}=19: 10: 10.6 ;$ Dec $=09: 05: 18$

and $\mathrm{DCO}^{+} / \mathrm{HCO}^{+}<0.07$. Based on the main isotopic $J=$ 4-3 transitions, the $\mathrm{HCN} / \mathrm{HCO}^{+}$column density ratio towards the SW clump is $\sim 7$ ( $\sim 4$ based on the ${ }^{13} \mathrm{C}$ lines $)$, similar to the values calculated for the source centre.

\subsubsection{The Eastern tail}

Figure 12 (left column) shows the observed line profiles towards the E-tail. Again, the lines are well-fitted by single Gaussian profiles, but the peak velocities of the lines are close to $0 \mathrm{~km} \mathrm{~s}^{-1}$, blue-shifted from the central source velocity. As discussed for the SW-clump above, we consider a range of physical conditions and find that column densities based on the HCN $J=$ 4-3 compared with the $J=3-2$ transition and the calculated $\mathrm{HCN} / \mathrm{HNC}$ ratio suggest that this gas is also at a lower temperature than the source centre.

We therefore use the same physical conditions for the E-tail as for the SW-clump: $T_{\text {kin }}=40 \mathrm{~K}$ and $n\left(\mathrm{H}_{2}\right)=5 \times$ $10^{5} \mathrm{~cm}^{-3}$. Table 6 lists fit parameters and resulting column densities. The $\mathrm{HCN} / \mathrm{HCO}^{+}$and $\mathrm{HCN} / \mathrm{HNC}$ ratios are very similar to those found for the SW-clump. The upper limit on the $\mathrm{DCO}^{+} / \mathrm{HCO}^{+}$ratio is more stringent and constrains $T_{\text {kin }}$ to $>25 \mathrm{~K}$. The $\mathrm{DCN} / \mathrm{HCN}$ ratio may be $0.02-0.04$, but the DCN $J=5-4$ detection is uncertain (see Fig. 12) as the line only covers two spectral channels and the signal-to-noise ratio is $<3$. Based on the rms noise in the DCN spectrum, using the integrated intensity for $\mathrm{HCN}$ and assuming a line width of $3 \mathrm{~km} \mathrm{~s}^{-1}$ for DCN as measured for $\mathrm{H}^{13} \mathrm{CN}$ and $\mathrm{H}^{13} \mathrm{CO}^{+}$, the $3 \sigma$ upper limit on the $\mathrm{DCN} / \mathrm{HCN}$ ratio is $\sim 3 \times 10^{-3}$. 
A\&A 525, A107 (2011)
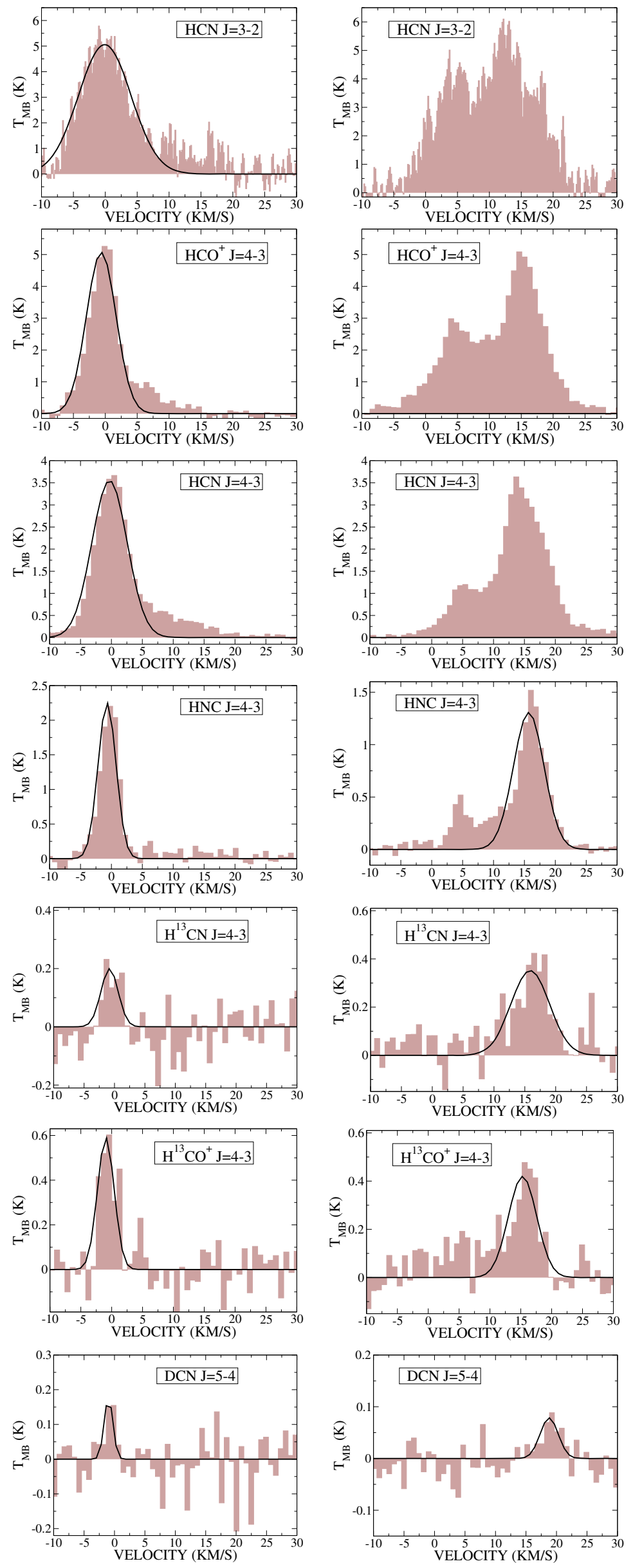

Fig. 12. Observed line profiles (shaded grey) and Gaussian fits (black lines) for the transitions observed towards the "East tail" (left) and the "North clump" (right) positions (RA = 19:10:16.6; Dec $=09: 05: 48$ and $\mathrm{RA}=$ 19:10:13.6; Dec = 09:06:48, respectively). 
Table 5. Estimated column densities towards the South-West clump.

\begin{tabular}{lccccccc}
\hline \hline Line & $\begin{array}{c}V_{\mathrm{LSR}} \\
\left(\mathrm{km} \mathrm{s}^{-1}\right)\end{array}$ & $\begin{array}{c}T_{\mathrm{mb}} \\
(\mathrm{K})\end{array}$ & $\begin{array}{c}F W H M \\
\left(\mathrm{~km} \mathrm{~s}^{-1}\right)\end{array}$ & $\begin{array}{c}\mathrm{rms} \\
(\mathrm{K})\end{array}$ & $\begin{array}{c}T_{\mathrm{ex}} \\
(\mathrm{K})\end{array}$ & $\tau$ & $\begin{array}{c}N \\
\left(10^{13} \mathrm{~cm}^{-2}\right)\end{array}$ \\
\hline $\mathrm{HCN} J=3-2$ & 9.4 & 6.1 & 9.5 & 0.52 & 11 & 4.9 & 33 \\
$\mathrm{HCN} J=4-3$ & 9.2 & 4.4 & 7.0 & 0.06 & 11 & 5.7 & 45 \\
$\mathrm{H}^{13} \mathrm{CN} J=4-3$ & 8.5 & 0.5 & 4.2 & 0.07 & 8.3 & 0.2 & 2.1 \\
$\mathrm{DCN} J=5-4$ & - & - & - & 0.04 & 10 & - & $<0.5$ \\
$\mathrm{HNC} J=4-3$ & 8.8 & 1.25 & 4.3 & 0.05 & 8.3 & 0.7 & 6.6 \\
$\mathrm{HCO}^{+} J=4-3$ & 9.3 & 5.0 & 7.3 & 0.12 & 13 & 1.4 & 6.6 \\
$\mathrm{H}^{13} \mathrm{CO}^{+} J=4-3$ & 9.0 & 0.8 & 4.2 & 0.08 & 12 & 0.2 & 0.5 \\
$\mathrm{DCO}^{+} J=5-4$ & - & - & - & 0.07 & 10 & - & $<0.5$ \\
\hline
\end{tabular}

Notes. Column densities are calculated with RADEX assuming $T_{\text {kin }}=40 \mathrm{~K}$ and $n\left(\mathrm{H}_{2}\right)=5 \times 10^{5} \mathrm{~cm}^{-3}$ towards the South-West clump at RA $=$ 19:10:10.6; Dec $=09: 05: 18$. We have based the column density ratios on the main lines of $\mathrm{HCN}$ and $\mathrm{HCO}^{+}$since the ${ }^{13} \mathrm{C}_{\text {-substituted spectra }}$ are rather noisy.

Table 6. Estimated column densities towards the Eastern tail.

\begin{tabular}{lccccccc}
\hline \hline Line & $\begin{array}{c}V_{\mathrm{LSR}} \\
\left(\mathrm{km} \mathrm{s}^{-1}\right)\end{array}$ & $\begin{array}{c}T_{\mathrm{mb}} \\
(\mathrm{K})\end{array}$ & $\begin{array}{c}F W H M \\
\left(\mathrm{~km} \mathrm{~s}^{-1}\right)\end{array}$ & $\begin{array}{c}\mathrm{rms} \\
(\mathrm{K})\end{array}$ & $\begin{array}{c}T_{\text {ex }} \\
(\mathrm{K})\end{array}$ & $\tau$ & $\begin{array}{c}N \\
\left(10^{13} \mathrm{~cm}^{-2}\right)\end{array}$ \\
\hline $\mathrm{HCN} J=3-2$ & -0.1 & 5.05 & 9.7 & 0.52 & 10 & 3.85 & 25.8 \\
$\mathrm{HCN} J=4-3$ & -0.3 & 3.55 & 6.7 & 0.04 & 9.5 & 4.01 & 33.0 \\
$\mathrm{H}^{13} \mathrm{CN} J=4-3$ & -0.8 & 0.20 & 3.4 & 0.08 & 8.5 & 0.07 & 0.7 \\
$\mathrm{DCN} J=5-4^{a}$ & -1.0 & 0.18 & 1.7 & 0.07 & 12 & 0.04 & 1.2 \\
$\mathrm{HNC} J=4-3$ & -0.7 & 2.25 & 3.4 & 0.08 & 8.6 & 1.7 & 9.9 \\
$\mathrm{HCO}^{+} J=4-3$ & -0.6 & 5.07 & 5.5 & 0.16 & 13 & 1.4 & 5.0 \\
$\mathrm{H}^{13} \mathrm{CO}^{+} J=4-3$ & -0.9 & 0.59 & 3.2 & 0.10 & 12 & 0.1 & 0.3 \\
$\mathrm{DCO}^{+} J=5-4$ & - & - & - & 0.06 & $\geq 10$. & - & $<0.2$ \\
\hline
\end{tabular}

Notes. Column densities are calculated with RADEX assuming $T_{\text {kin }}=40 \mathrm{~K}$ and $n\left(\mathrm{H}_{2}\right)=5 \times 10^{5} \mathrm{~cm}^{-3}$ towards the Eastern tail at RA = 19:10:16.6; $\mathrm{Dec}=09: 05: 48$. We show column density ratios based on both the main lines of $\mathrm{HCN}$ and $\mathrm{HCO}^{+}$and the ${ }^{13} \mathrm{C}$-substituted lines, since there is some excess emission at higher velocities in the main $\mathrm{HCN}$ and $\mathrm{HCO}^{+}$lines that has not been fitted (see Fig. 12).

(a) Tentative detection based on the rms noise in the spectrum, $N(\mathrm{DCN})<4 \times 10^{12} \mathrm{~cm}^{-2}$ for $T_{\mathrm{ex}} \geq 10 \mathrm{~K}$ and DCN/HCN $<0.002$.

\subsubsection{The Northern clump}

The line profiles of the strongest lines towards the N-clump are double peaked, but in contrast to the source centre, the red-shifted peaks are stronger than the blue-shifted peaks (see Fig. 12; right column). These line profiles are not consistent with spherical infall motions. Since the isotopic species peak closely in velocity to the redshifted emission, the bulk of the gas appears to be at this velocity. The blueshifted peak is probably due to a bipolar outflow or other unresolved kinematic substructure.

We have fitted single Gaussian profiles to the detected lines that are not strongly self-absorbed $\left(\mathrm{HNC}, \mathrm{H}^{13} \mathrm{CN}\right.$, and $\mathrm{H}^{13} \mathrm{CO}^{+} J=4-3$ and DCN $J=5-4$; though the signal-tonoise ratio of the DCN line is $<2$ ) and find that these peak at $15-16 \mathrm{~km} \mathrm{~s}^{-1}\left(\sim 19 \mathrm{~km} \mathrm{~s}^{-1}\right.$ for DCN $\left.J=5-4\right)$. The calculated $N(\mathrm{HCN}) / N(\mathrm{HNC})$ ratio, based on the $\mathrm{H}^{13} \mathrm{CN}$ observation, is $\sim 20$, which is between the values for the source centre $(\sim 50)$ and the two clumps (5-7). We therefore adopt an intermediate value for the kinetic temperature (see also Sect. 4.2). Note that our fit to the HNC line profile is dominated by the red-shifted peak and thus underestimates the HNC column density, but only by $\sim 10 \%$, which does not change our result.

Table 7 lists the fit parameters and resulting column densities for $T_{\text {kin }}=75 \mathrm{~K}$ and $n\left(\mathrm{H}_{2}\right)=5 \times 10^{5} \mathrm{~cm}^{-3}$. The calculated $\mathrm{DCN} / \mathrm{HCN}$ ratio is an order of magnitude lower than towards the E-tail, while the $\mathrm{HCN} / \mathrm{HCO}^{+}$ratio is $\geq 1.5$ times higher than towards the other regions we looked at.

\section{Discussion}

We have presented velocity-resolved maps of the W49A region in $\mathrm{HCN}, \mathrm{HNC}, \mathrm{HCO}^{+}$, and isotopic line emission over a $2^{\prime}(6.6 \mathrm{pc})$ field at $15^{\prime \prime}(0.83 \mathrm{pc})$ resolution. The $\mathrm{HCN}$ and $\mathrm{HCO}^{+}$emission extends over $60-100^{\prime \prime}$, while $\mathrm{HNC}, \mathrm{H}^{13} \mathrm{CN}$ and $\mathrm{H}^{13} \mathrm{CO}^{+}$emission is confined to $\sim 40^{\prime \prime}$ and rarer isotopes are only seen at the source centre. The maps show a molecular "core", elongated East-West, with "tails" to the North and East, and a separate "clump" to the South-West. While most of the rare isotopic species show single-peaked velocity profiles, the main $\mathrm{HCN}$ and $\mathrm{HCO}^{+}$species show double-peaked, asymmetric profiles suggestive of infall around the peak of the emission.

Table 8 summarizes our adopted physical conditions and derived column densities for the various gas components in the W49A region. The $\mathrm{HCN} / \mathrm{HCO}^{+}$column density ratio is seen to vary from $\approx 3$ in the SW clump and $\mathrm{E}$ tail to $\approx 10$ in the core and the $\mathrm{N}$ clump. The $\mathrm{HCN} / \mathrm{HNC}$ column density ratio is generally higher and varies from $\approx 6$ in the $\mathrm{E}$ tail to $\approx 20$ in the other gas components. The $\mathrm{HCN} / \mathrm{HCO}^{+}$column density ratios are quite different from the observed line ratios which range from 0.3 to 1.4 , while the isotopic line ratio $\mathrm{H}^{13} \mathrm{CN} / \mathrm{H}^{13} \mathrm{CO}^{+}$of $0.3-5.3$ is closer to the column density ratio.

The HNC line has a similar central velocity and line width as the $\mathrm{H}^{13} \mathrm{CO}^{+}$and $\mathrm{H}^{13} \mathrm{CN}$ lines, which is considerably different from the main $\mathrm{HCN}$ and $\mathrm{HCO}^{+}$lines. The $\mathrm{HNC}$ line thus seems to trace the inner core regions more than $\mathrm{HCN}$ and $\mathrm{HCO}^{+}$. This difference may be due to a low HNC abundance in the warm 
Table 7. Estimated column densities towards the Northern clump.

\begin{tabular}{lccccccc}
\hline \hline Molecule & $\begin{array}{c}V_{\mathrm{LSR}} \\
\left(\mathrm{km} \mathrm{s}^{-1}\right)\end{array}$ & $\begin{array}{c}T_{\mathrm{mb}} \\
(\mathrm{K})\end{array}$ & $\begin{array}{c}F W H M \\
\left(\mathrm{~km} \mathrm{~s}^{-1}\right)\end{array}$ & $\begin{array}{c}\mathrm{rms} \\
(\mathrm{K})\end{array}$ & $\begin{array}{c}T_{\mathrm{ex}} \\
(\mathrm{K})\end{array}$ & $\tau$ & $\begin{array}{c}N \\
\left(10^{12} \mathrm{~cm}^{-2}\right)\end{array}$ \\
\hline $\mathrm{H}^{13} \mathrm{CN} J=4-3$ & 15.9 & 0.35 & 7.4 & 0.06 & 10 & 0.09 & 13.8 \\
$\mathrm{DCN} J=5-4^{a}$ & 18.8 & 0.08 & 3.5 & 0.05 & 14 & 0.01 & 4.3 \\
$\mathrm{HNC} J=4-3$ & 15.7 & 1.31 & 5.8 & 0.05 & 10 & 0.38 & 46.8 \\
$\mathrm{H}^{13} \mathrm{CO}^{+} J=4-3$ & 15.3 & 0.42 & 5.4 & 0.07 & 15 & 0.04 & 0.9 \\
$\mathrm{DCO}^{+} J=5-4$ & - & - & - & 0.06 & 15 & - & $<0.7$ \\
\hline
\end{tabular}

Notes. Column densities are calculated with RADEX assuming $T_{\text {kin }}=75 \mathrm{~K}$ and $n\left(\mathrm{H}_{2}\right)=5 \times 10^{5} \mathrm{~cm}^{-3}$ towards RA = 19:10:13.6; Dec $=09: 06: 48$. (a) Tentative detection based on the rms noise in the spectrum, $N(\mathrm{DCN})<3.7 \times 10^{12} \mathrm{~cm}^{-2}$ for $T_{\mathrm{ex}} \geq 10 \mathrm{~K}$ and $\mathrm{DCN} / \mathrm{HCN}<0.008$.

Table 8. Summary of adopted physical conditions and derived column densities for the various gas components in the W49A region.

\begin{tabular}{lccccc}
\hline \hline Component & $T_{\text {kin }}$ & $n\left(\mathrm{H}_{2}\right)$ & \multicolumn{3}{c}{$N\left(10^{14} \mathrm{~cm}^{-2}\right)$} \\
& $(\mathrm{K})$ & $\left(10^{5} \mathrm{~cm}^{-3}\right)$ & $\mathrm{HCN}$ & $\mathrm{HCO}^{+}$ & $\mathrm{HNC}$ \\
\hline Core & 100 & 20 & $20-70$ & $2-10$ & $\approx 1$ \\
SW clump & 40 & 5 & 10 & 4 & 0.7 \\
E tail & 40 & 5 & 6 & 2 & 1 \\
N clump & 75 & 5 & 10 & 0.7 & 0.5 \\
\hline
\end{tabular}

central gas, but a possible difference in critical density between $\mathrm{HCN}$ and $\mathrm{HCO}^{+}$on one hand and $\mathrm{HNC}$ on the other may also play a role (Sarrasin et al. 2010).

Since the $\mathrm{HCN}, \mathrm{HNC}$ and $\mathrm{HCO}^{+}$main isotopic lines are optically thick, the observed line intensity is not a direct measure of column density, but is rather the product of surface area, filling factor and temperature. The cloud structure of W49 is not homogeneous, as shown by the difference in line width between the main and rare isotopic lines, and by the main beam brightness of the lines which is well below the estimated kinetic temperature. A possible geometry, commonly invoked to explain the large-scale CO emission from Giant Molecular Clouds, is an ensemble of small gas clumps in the beam. The inhomogeneous source structure does not directly affect our estimates of the beam-averaged column density, which are based on rare isotopic lines. The column densities are indirectly affected through our estimate of the volume density, which influences the fraction of $\mathrm{HCN}$ in the $J=4$ state.

We now discuss our derived column densities toward W49A by comparing them to predictions from physical and chemical models (Sects. 4.1 and 4.2) and to the results of extragalactic observations (Sect. 4.3).

\subsection{Comparison to $P D R / X D R$ models}

One interesting comparison which these observations allow are with the models by Meijerink et al. (2007) who predict the molecular line emission from irradiated dense gas in galaxies using models of PDRs (photon dominated regions) and XDRs (X-ray dominated regions). Specifically Meijerink et al. predict the intensity ratios in the $J=1-0$ and $J=4-3$ transitions of $\mathrm{HCN}, \mathrm{HCO}^{+}$and HNC. The measured velocity-integrated values of these ratios towards W49A are summarised in Table 2 where the values for the $J=1-0$ transition come from maps made with the IRAM 30 m telescope by Peng et al. (2007) and Peng et al. (in prep.).

The observed $\mathrm{HCN} / \mathrm{HCO}^{+}$ratios in both the $J=1-0$ and $J=4-3$ transitions are inconsistent with all the Meijerink et al. XDR models, but are consistent with emission from a PDR. The
$J=1-0$ line ratio implies a well constrained number density of $n \sim 4 \times 10^{4} \mathrm{~cm}^{-3}$, consistent with the ratio in the $J=4-3$ transitions. However, the PDR model has difficulties explaining our observed $\mathrm{HNC} / \mathrm{HCN}$ ratios. Our observed $\mathrm{HNC} / \mathrm{HCN}$ ratio in the $J=1-0$ line would imply number densities $>3 \times 10^{5} \mathrm{~cm}^{-3}$, while the $J=4-3$ transitions imply $n<2 \times 10^{4} \mathrm{~cm}^{-3}$. The $\mathrm{HNC} / \mathrm{HCN}$ line ratios are better explained by the XDR model, where both transitions are consistent with densities between $10^{4} \mathrm{~cm}^{-3}$ and $\sim 10^{5} \mathrm{~cm}^{-3}$ but require X-ray fields with fluxes $>5 \mathrm{erg} \mathrm{cm}^{-2} \mathrm{~s}^{-1}$ at the lowest densities up to $>100 \mathrm{erg} \mathrm{cm}^{-2} \mathrm{~s}^{-1}$ at the highest densities. We conclude that no single PDR or XDR model is able to reproduce our observations, so that a more complex, multi-component model must be invoked. One caveat is the Meijerink et al. models should be updated with the new collision data for HCN and HNC (Sarrasin et al. 2010).

\subsection{Comparison to chemical models}

We also compare the observed column density ratios with predictions from kinetic models of interstellar chemistry. The models consider 340 species linked by $>10000$ reactions with rate co-efficients based on the latest release of the UMIST Database for Astrochemistry, RATE06 ${ }^{1}$ (Woodall et al. 2007). The chemical reaction set has been extended to include deuterated analogues of all hydrogen-containing species (see e.g. Roberts \& Millar 2000). The models are "pseudo-time-dependent", in that the physical conditions remain constant over time and they consider only gas-phase chemical reactions (with the exception of $\mathrm{H}_{2}, \mathrm{HD}$, and $\mathrm{D}_{2}$ formation on grain-surfaces). Figure 13 shows steady-state fractional abundances and abundance ratios predicted by a set of models run with $n\left(\mathrm{H}_{2}\right)=10^{6} \mathrm{~cm}^{-3}$ and values for $T_{\text {kin }}$ between 10 and $200 \mathrm{~K}$.

As discussed in Sect. 1, the HCN/HNC ratio increases and the molecular $\mathrm{D} / \mathrm{H}$ ratios decrease as $T_{\text {kin }}$ increases. The fractional abundances of $\mathrm{HCN}$ and $\mathrm{HNC}$ fall with increasing $T_{\text {kin }}$, while the abundance of $\mathrm{HCO}^{+}$increases. We expect, therefore, that the $\mathrm{HCN} / \mathrm{HCO}^{+}$ratio will also trace the gas temperature.

The $\mathrm{HCN} / \mathrm{HNC}$ ratios we measure in W49A vary between 50 at the source centre to 20 towards the N-clump and 6-7 in the SW-clump and E-tail. It is these ratios that we have mainly used to constrain the temperatures of these regions as 100, 75, and $40 \mathrm{~K}$, respectively. In the future, we plan to use the SLS data to put more direct constraints on the gas temperature and density across the $\mathrm{W} 49 \mathrm{~A}$ region.

The $\mathrm{HCN} / \mathrm{HCO}^{+}$ratios we observe, however, are between 3 and 15 . The steady-state chemical models predict $\mathrm{HCN}>\mathrm{HCO}^{+}$ only for $T_{\text {kin }}<25 \mathrm{~K}$. Presumably, though, the source formed from a molecular cloud where the gas-phase abundances had

$\overline{1 \text { http://www .udfa.net }}$ 

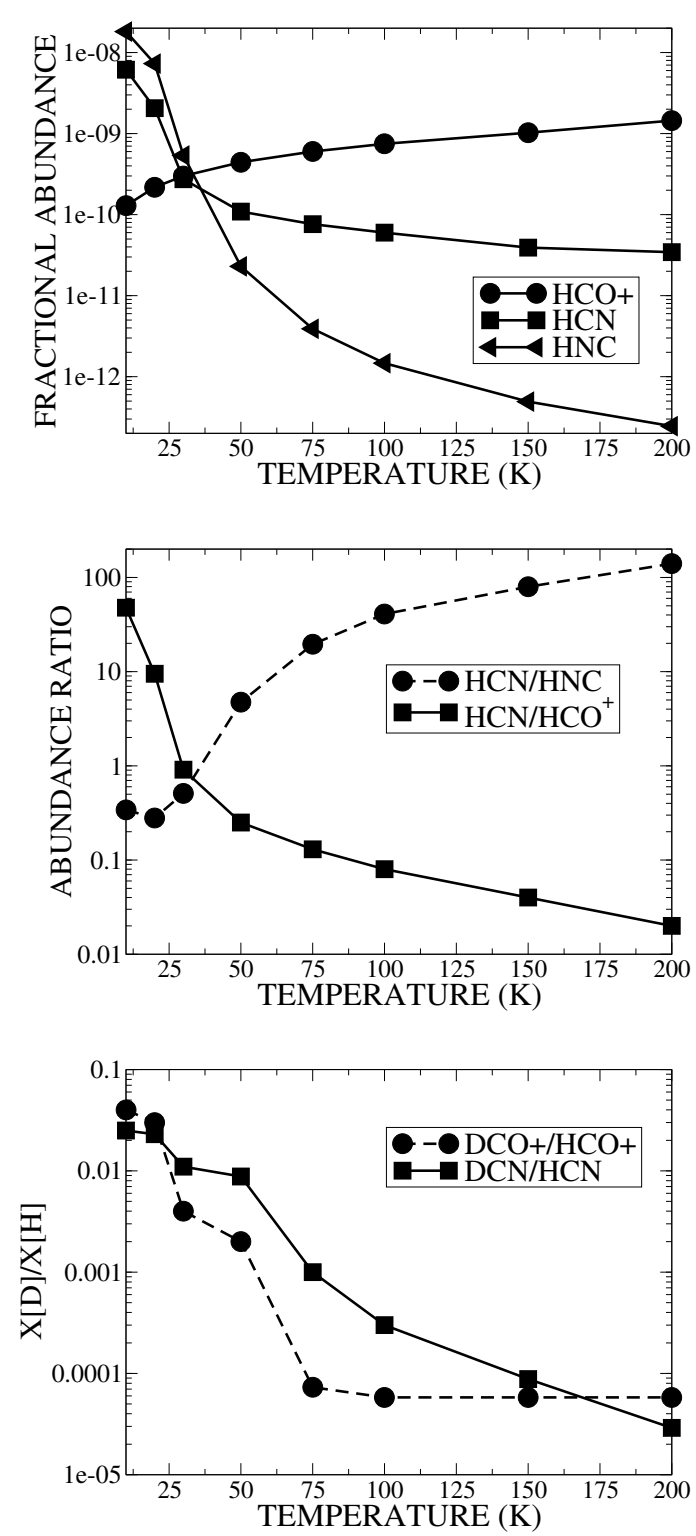

Fig. 13. Steady-state fractional abundances and abundance ratios predicted by gas-phase chemical models run at different temperatures; $n\left(\mathrm{H}_{2}\right)=10^{6} \mathrm{~cm}^{-3}$.

equilibrated at a much lower temperature. If we use the steadystate abundances at $10 \mathrm{~K}$ as the initial abundances for the models at higher temperatures it takes $>10^{5}$ years for the chemistry to reach a new steady-state. This is larger than the expected lifetimes of hot-cores in high mass star forming regions $\left(3-5 \times 10^{4} \mathrm{yr}\right.$ : Wilner et al. 2001).

Figure 14 shows the evolution of the $\mathrm{HCN} / \mathrm{HCO}^{+}$and $\mathrm{HCN} / \mathrm{HNC}$ abundance ratios from gas which has reached steady state at $10 \mathrm{~K}$ at time $=0 \mathrm{yr}$ and is then heated to 40 or $100 \mathrm{~K}$. While the $\mathrm{HCN} / \mathrm{HCO}^{+}$ratio maintains its "low-temperature" value, $>1$, for $\geq 10^{4} \mathrm{yr}$, the $\mathrm{HCN} / \mathrm{HNC}$ ratio varies much more with time, increasing to $\sim 10 \times$ its "high temperature" value before decreasing to steady-state. It is not really possible from these simple models to put an absolute age on the W49A core, as the timescales do depend on the density and on the rate of warming of the gas, but they could explain why the observed $\mathrm{HCN} / \mathrm{HNC}$ ratios better probe the current gas temperature, while the $\mathrm{HCN} / \mathrm{HCO}^{+}$ratios are closer to what we expect in colder gas.
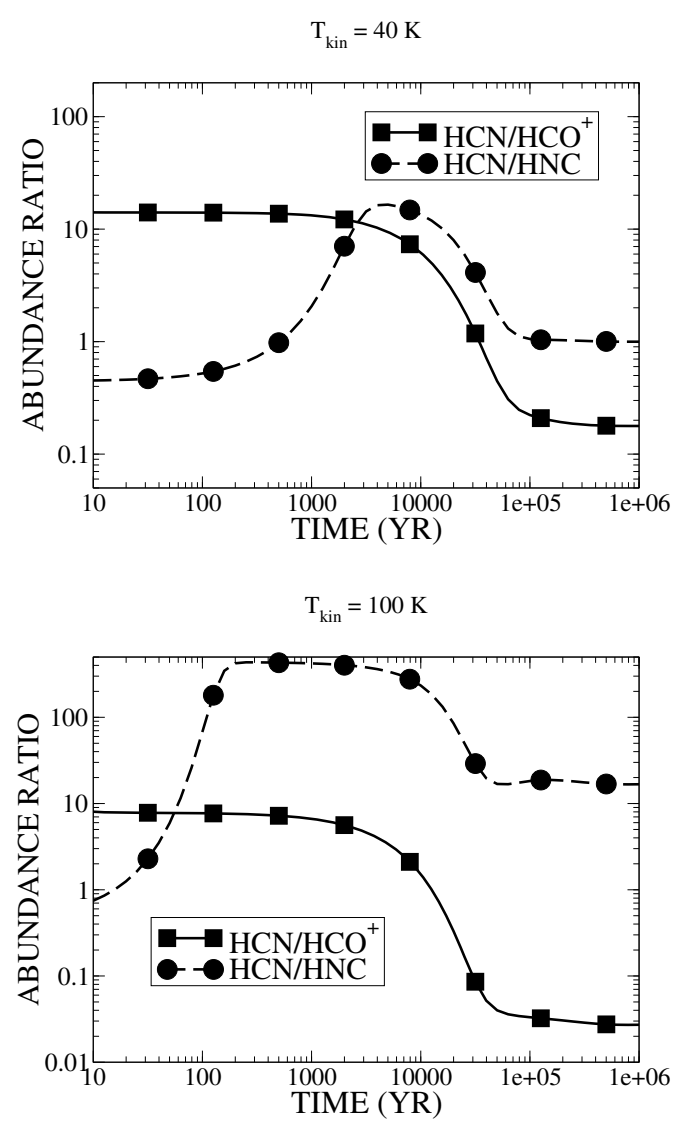

Fig. 14. Fractional abundance ratios varying over time from gas-phase chemical models assuming $n\left(\mathrm{H}_{2}\right)=10^{6} \mathrm{~cm}^{-3}$. The initial abundances come from a steady-state model at $10 \mathrm{~K}$; the kinetic temperatures are $40 \mathrm{~K}($ top $)$ and $100 \mathrm{~K}$ (bottom).

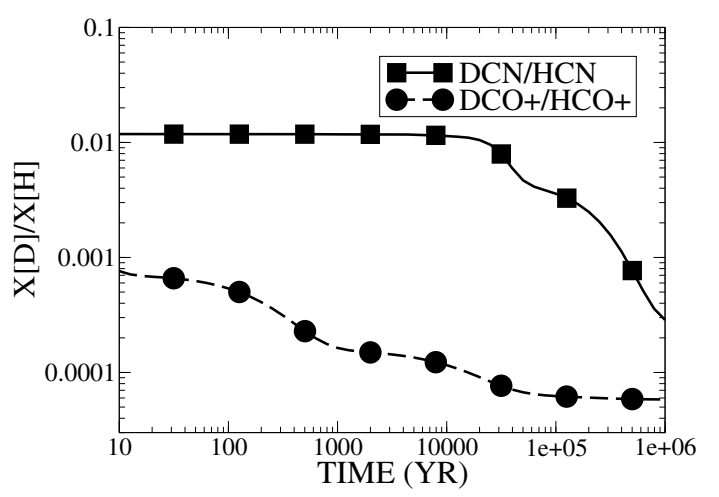

Fig. 15. Fractional abundance ratios varying over time from a gas-phase chemical models assuming $n\left(\mathrm{H}_{2}\right)=10^{6} \mathrm{~cm}^{-3}$ and $T_{\text {kin }}=100 \mathrm{~K}$. The initial abundances come from a steady-state model at $10 \mathrm{~K}$.

Similarly, for the DCN/HCN ratio, Fig. 15 shows that it also takes $>10^{4} \mathrm{yr}$ at $100 \mathrm{~K}$ for the fractionation set at low temperatures to begin to fall (see Sect. 1). In contrast, the $\mathrm{DCO}^{+} / \mathrm{HCO}^{+}$ratio resets to a "high-temperature" value within 1000 yr. Thus, the $\mathrm{DCN} / \mathrm{HCN}$ ratios of $4 \times 10^{-4}-10^{-3}$ observed towards the centre of W49A (and the tentative estimate of $\mathrm{DCN} / \mathrm{HCN}=0.02-0.04$ in the E-tail at $40 \mathrm{~K}$ ) are not inconsistent with current higher kinetic temperatures as, like the 
A\&A 525, A107 (2011)

Table 9. Observed line ratios in selected extragalactic objects and comparison to W49A.

\begin{tabular}{lcccccc}
\hline \hline Ratio & APM 08279 & NGC 253 & \multicolumn{4}{c}{ W49A } \\
& & & Centre & North & East & Southwest \\
\hline $\mathrm{HCN} / \mathrm{HCO}^{+} 4-3^{a}$ & 0.98 & 1.03 & 0.5 & 0.6 & 0.9 & 0.8 \\
$\mathrm{H}^{13} \mathrm{CN} / \mathrm{H}^{13} \mathrm{CO}^{+} 2-1$ & - & $1.66-3.72^{b}$ & $\approx 5^{c}$ & $\approx 5^{c}$ & $\approx 1.4^{c}$ & $\approx 1.4^{c}$ \\
$\mathrm{HCN} 4-3^{a} / \mathrm{HNC} 1-0$ & 1.57 & $0.03-0.14^{b}$ & 2.0 & 1.0 & 0.9 & 1.4 \\
$\mathrm{H}^{13} \mathrm{CN} / \mathrm{HN}^{13} \mathrm{C} 2-1$ & - & 2.29 & & & & \\
$\mathrm{HCN} 4-3 / \mathrm{H}^{13} \mathrm{CN} 2-1$ & - & $0.42-0.56^{b}$ & & & & \\
$\mathrm{HCO}^{+} 4-3 / \mathrm{H}^{13} \mathrm{CO}^{+} 2-1$ & - & $0.90-1.51^{b}$ & & & & \\
$\mathrm{HCO}^{+} 4-3 / \mathrm{HC}^{18} \mathrm{O}^{+} 2-1$ & - & 2.60 & & & & \\
$\mathrm{DNC} 2-1 / \mathrm{HNC} 1-0$ & - & $0.01-0.05^{b}$ & & & & \\
\hline
\end{tabular}

Notes. ${ }^{(a)} J=5-4$ for APM 08279; ${ }^{(b)}$ values for the two observed velocity components; ${ }^{(c)}$ estimated from the 4-3 ratio (see text).

$\mathrm{HCN} / \mathrm{HCO}^{+}$ratio, the fractionation may be preserved from an earlier, colder phase of the core's evolution.

Of course, the chemistry in W49A is unlikely to be this simple. For each line of sight we may be looking through multiple gas-components with different physical conditions (certainly, at the source centre the JCMT beam covers several embedded hot-core sources; see Fig. 1). The gas-phase chemical models also assume that the density and, therefore, the visual extinction are high enough that photo-reactions are unimportant. This is supported by the IRAC $8 \mu \mathrm{m}$ image of W49A (Peng et al. 2010) not showing maxima at any of our "clumps" and "tails", which would be indicative of a PDR.

\subsection{Comparison to extragalactic regions}

Both $\mathrm{HCN}$ and $\mathrm{HCO}^{+}$have been advocated as tracers of dense gas in external galaxies (Baan et al. 2008; Graciá-Carpio et al. 2006), but to date the majority of extragalactic observations have focussed on the $J=1-0$ transitions of these species. However, due to their higher critial densities and higher upper energy levels, the higher $J$ transitions are likely to be more efficient probes of the high density, warm gas associated with star formation. In addition, the comparison with lower energy transitions provides a probe of the (range of) excitation conditions in the gas (Papadopoulos 2007). A survey of nearby active galaxies in the $J=1-0$ to $J=3-2$ transitions of both HCN and $\mathrm{HCO}^{+}$shows that the line intensity from higher $J$ transitions relative to lower transitions increases in increasingly starburstdominated galaxies, as does the ratio of $\mathrm{HCO}^{+}$to $\mathrm{HCN}$ emission in a particular $J$ transition (Krips et al. 2008). The temperature $(50-100 \mathrm{~K})$, density $\left((0.5-2) \times 10^{6} \mathrm{~cm}^{-3}\right)$ and column density $\left(10^{14}-10^{15} \mathrm{~cm}^{-2}\right)$ properties of W49A on the scales observed here are similar to those derived by Krips et al. (2008), indicating that W49A may be a valuable analogue for understanding the emission from other galaxies. Consistent with the observations of Krips et al. (2008), the strength of the $\mathrm{HCO}^{+} J=4-3$ transition in W49A suggests that this line should be a strong and robust tracer of the dense gas in extragalactic star forming regions. One advantage of the 4-3 line over the 1-0 line is that it is much less affected by absorption in low-density foreground layers.

The $J=5-4$ lines of $\mathrm{HCN}, \mathrm{HCO}^{+}$and $\mathrm{HNC}$ have been observed for the high- $z$ galaxy APM 08279+5255 (Guélin et al. 2007). In NGC 253, several isotopologues of HCN, HNC and $\mathrm{HCO}^{+}$have also been observed (Hüttemeister et al. 1995; Wagg et al. 2005; García-Burillo et al. 2006; Martín et al. 2006). Estimates of ratios derived from these studies are presented in Table 9. These studies make these two very active sources particularly relevant to compare to the properties derived for each component identified in W49A (Sects. 3.3.1-3.3.4).

The comparison between APM 08279+5255 and the components identified in W49A is limited by the number of ratios available for the high- $z$ source. In contrast, the data for NGC 253 are much more complete than those for W49A. Concentrating on those ratios where data exist for all sources, we note the following from the data in Table 9. First, the $\mathrm{HCN} / \mathrm{HCO}^{+} 4-3$ ratios in the East and Southwest regions are similar to those in APM 08279 and NGC 253, whereas the values in the Centre and North regions are lower. The minimum value of this ratio measured in the entire $\mathrm{W} 49 \mathrm{~A}$ region is $\approx 0.3$. Second, the $\mathrm{HCN} 4-3 / \mathrm{HNC} 1-0$ ratios at all positions in W49A are much higher than measured toward NGC 253, while the values toward the Centre and North regions bracket the ratio measured toward APM 08279. Third, the $\mathrm{H}^{13} \mathrm{CN} / \mathrm{H}^{13} \mathrm{CO}^{+} 2-1$ ratios, which are based on the observed 4-3 ratios, using RADEX at the temperature and density of the region to convert the 4-3 ratio to the 2-1 ratio, bracket the value measured toward NGC 253. Interestingly, there is little evidence that the fact that APM 08279 is hosting a quasar is making any impact on the emission in our observed lines.

Based on the similarity of the line ratios and the column densities, we conclude that W49A appears to be a reasonable model for extragalactic star-forming regions.

\section{Conclusions}

We have mapped $\mathrm{HCN}, \mathrm{HNC}, \mathrm{HCO}^{+}$, and related isotopologues in the luminous star-forming region W49A. Line ratios of these dense gas tracers are often used to probe the chemical state and ionisation processes in external galaxies. We find that the $\mathrm{HCN} / \mathrm{HCO}^{+} J=4-3$ line ratios are $<1$ across most of the source, but that these lines, particularly $\mathrm{HCO}^{+}$, are highly optically thick; the $\mathrm{H}^{13} \mathrm{CN} / \mathrm{H}^{13} \mathrm{CO}^{+} J=4-3$ line ratios are $\geq 1$ towards the source centre and slightly higher just to the northwest; the $\mathrm{HCN} / \mathrm{HCO}^{+}$column density ratios are $3-10$. The $\mathrm{HCN} / \mathrm{HNC}$ column density ratio varies from $\approx 6$ in the Eastern tail to $\approx 20$ in the other gas components.

We also find that, although there are embedded hot cores towards the centre of W49A which are not resolved in these observations, column densities towards this region are consistent with an average gas temperature of $\sim 100 \mathrm{~K}$ and density $>10^{6} \mathrm{~cm}^{-3}$. There appear to be several cooler $\left(T_{\text {kin }} \sim 40 \mathrm{~K}\right)$ but still relatively dense $\left(n\left(\mathrm{H}_{2}\right)>10^{5} \mathrm{~cm}^{-3}\right)$ clumps of gas surrounding, and possibly infalling onto, the source centre.

Comparison of our observations with the PDR and XDR models of Meijerink et al. (2007) shows contradictory 
results. While the $\mathrm{HCN}$ and $\mathrm{HCO}^{+}$emission could be consistent with a PDR, this is inconsistent with the $\mathrm{HNC}$ to $\mathrm{HCN}$ ratio which points to emisssion from a XDR. We interpret this discrepancy as evidence that irradiation plays only a minor part in the chemistry of the molecular gas in W49A.

We also find that regions of W49A have significant similarities to observations of the starburst galaxy NGC 253 and the quasar host APM 08279. This agreement seems to imply that over scales of a few $100 \mathrm{pc}$, the line emission from external galaxies is not dominated by hot core physics but rather by extended warm gas-phase chemistry. The W49A region thus may be a good template for starburst galaxies, and the current data indicate that irradiation by an AGN has a limited effect on the chemistry of the surrounding molecular gas.

The observations discussed here highlight the potential problems in the interpretation of extragalactic systems on the basis of a few transitions of a limited set of molecular species. The extragalactic star forming regions which such observations probe are likely at least as complex and structured as W49A. A better understanding of the origin of the emission towards W49A and the internal physical and kinematic structure of the source will require velocity resolved, high angular resolution observations with instruments such as the SMA and ALMA interferometers. These observations will be able to address the origin of the velocity structure seen in the JCMT observations as well as determine whether different components of the emission are spatially segregated due to differences in excitation or chemistry. Together with the analysis of the more complete set of molecular species probing other physical and chemical environments which will be available in the full JCMT SLS-observations of W49A and the Orion Bar (Van der Wiel et al. 2009), such studies of W49A can provide more insight into the relationship between the molecular emission, the structure and the star formation in star forming regions in other galaxies.

Acknowledgements. The authors would like to thank all members of the SLS consortium for carrying out the JCMT Legacy survey observations, Libby Miller (Belfast) for reducing the JCMT $230 \mathrm{GHz}$ data, Jenny Williams (Manchester) for reducing the SCUBA data, Zsófia Nagy (Groningen) for reducing the HNC data, and Tzu-Cheng Peng \& Friedrich Wyrowski (Bonn) for making their IRAM 30m maps available to us ahead of publication. The James Clerk Maxwell Telescope is operated by the Joint Astronomy Centre on behalf of the Science and Technology Facilities Council of the United Kingdom, The Netherlands Organisation for Scientific Research, and the National Research Council of Canada. Astrophysics at Queens University Belfast and The Jodrell Bank Centre for Astrophysics is supported by STFC. E.B. acknowledges support from an STFC grant. We thank the referee, Paul Ho, and the editor, Malcolm Walmsley, for their useful comments on the manuscript.

\section{References}

Aalto, S., Monje, R., \& Martín, S. 2007, A\&A, 475, 479

Baan, W. A., Henkel, C., Loenen, A. F., Baudry, A., \& Wiklind, T. 2008, A\&A, 477, 747

Bayet, E., Viti, S., Williams, D. A., \& Rawlings, J. M. C. 2008, ApJ, 676, 978

Bayet, E., Aladro, R., Martín, S., Viti, S., \& Martín-Pintado, J. 2009a, ApJ, 707, 126

Bayet, E., Gerin, M., Phillips, T. G., \& Contursi, A. 2009b, MNRAS, 399, 264

Buckle, J. V., Hills, R. E., Smith, H., et al. 2009, MNRAS, 399, 1026

Dickel, H. R., \& Auer, L. H. 1994, ApJ, 437, 222
Dickel, H. R., Williams, J. A., Upham, D. E., et al. 1999, ApJS, 125, 413

Dumouchel, F., Faure, A., \& Lique, F. 2010, MNRAS, 406, 2488

Gao, Y., \& Solomon, P. M. 2004, ApJ, 606, 271

García-Burillo, S., Graciá-Carpio, J., Guélin, M., et al. 2006, ApJ, 645, L17

Goldsmith, P. F., Langer, W. D., Ellder, J., Kollberg, E., \& Irvine, W. 1981, ApJ, 249,524

Goldsmith, P. F., Irvine, W. M., Hjalmarson, A., \& Ellder, J. 1986, ApJ, 310, 383

Graciá-Carpio, J., García-Burillo, S., Planesas, P., \& Colina, L. 2006, ApJ, 640, L135

Greve, T. R., Papadopoulos, P. P., Gao, Y., \& Radford, S. J. E. 2009, ApJ, 692, 1432

Guélin, M., Salomé, P., Neri, R., et al. 2007, A\&A, 462, L45

Gwinn, C. R., Moran, J. M., \& Reid, M. J. 1992, ApJ, 393, 149

Hatchell, J., Millar, T. J., \& Rodgers, S. D. 1998, A\&A, 332, 695

Hüttemeister, S., Henkel, C., Mauersberger, R., et al. 1995, A\&A, 295, 571

Jaffe, D. T., Harris, A. I., \& Genzel, R. 1987, ApJ, 316, 231

Kohno, K. 2005, in The Evolution of Starbursts, ed. S. Hüttmeister, E. Manthey, D. Bomans, \& K. Weis, Am. Inst. Phys. Conf. Ser., 783, 203

Kramer, C., Aalto, S., \& Simon, R. 2008, Far-Infrared Workshop 2007, EAS Publications Series, 31

Krips, M., Neri, R., García-Burillo, S., et al. 2008, ApJ, 677, 262

Krumholz, M. R., \& Thompson, T. A. 2007, ApJ, 669, 289

Linsky, J. L. 2006, in Astrophysics in the Far Ultraviolet: Five Years of Discovery with FUSE, ed. G. Sonneborn, H. W. Moos, \& B.-G. Andersson, ASP Conf. Ser., 348, 70

Loinard, L., Castets, A., Ceccarelli, C., et al. 2002, Planet. Space Sci., 50, 1205

Martín, S., Mauersberger, R., Martín-Pintado, J., Henkel, C., \& García-Burillo, S. 2006, ApJS, 164, 450

Meijerink, R., Spaans, M., \& Israel, F. P. 2007, A\&A, 461, 793

Millar, T. J., Bennett, A., \& Herbst, E. 1989, ApJ, 340, 906

Motte, F., Schilke, P., \& Lis, D. C. 2003, ApJ, 582, 277

Mufson, S. L., \& Liszt, H. S. 1977, ApJ, 212, 664

Narayanan, D., Cox, T. J., Shirley, Y., et al. 2008, ApJ, 684, 996

Papadopoulos, P. P. 2007, ApJ, 656, 792

Parise, B., Ceccarelli, C., Tielens, A. G. G. M., et al. 2002, A\&A, 393, L49

Peng, T.-C., Wyrowski, F., van der Tak, F., et al. 2007, in Molecules in Space and Laboratory

Peng, T., Wyrowski, F., van der Tak, F. F. S., Menten, K. M., \& Walmsley, C. M. 2010, A\&A, 520, A84

Plume, R., Kaufman, M. J., Neufeld, D. A., et al. 2004, ApJ, 605, 247

Plume, R., Fuller, G. A., Helmich, F., et al. 2007, PASP, 119, 102

Riechers, D. A., Walter, F., Carilli, C. L., et al. 2006, ApJ, 645, L13

Roberts, H., \& Millar, T. J. 2000, A\&A, 361, 388

Roberts, H., \& Millar, T. J. 2007, A\&A, 471, 849

Rodgers, S. D., \& Millar, T. J. 1996, MNRAS, 280, 1046

Sarrasin, E., Abdallah, D. B., Wernli, M., et al. 2010, MNRAS, 404, 518

Schilke, P., Walmsley, C. M., Pineau des Forêts, G., et al. 1992, A\&A, 256, 595

Schöier, F. L., van der Tak, F. F. S., van Dishoeck, E. F., \& Black, J. H. 2005, A\&A, 432, 369

Serabyn, E., Guesten, R., \& Schulz, A. 1993, ApJ, 413, 571

Simon, R., Jackson, J. M., Clemens, D. P., Bania, T. M., \& Heyer, M. H. 2001, ApJ, 551, 747

Van der Tak, F. F. S., Black, J. H., Schöier, F. L., Jansen, D. J., \& van Dishoeck, E. F. 2007, A\&A, 468, 627

Van der Wiel, M. H. D., van der Tak, F. F. S., Ossenkopf, V., et al. 2009, A\&A, 498, 161

Vastel, C., Phillips, T. G., Ceccarelli, C., \& Pearson, J. 2003, ApJ, 593, L97

Wagg, J., Wilner, D. J., Neri, R., Downes, D., \& Wiklind, T. 2005, ApJ, 634, L13

Ward-Thompson, D., \& Robson, E. I. 1990, MNRAS, 244, 458

Watson, W. D. 1976, Rev. Mod. Phys., 48, 513

Welch, W. J., Dreher, J. W., Jackson, J. M., Terebey, S., \& Vogel, S. N. 1987, Science, 238, 1550

Wilner, D. J., De Pree, C. G., Welch, W. J., \& Goss, W. M. 2001, ApJ, 550, L81

Wilson, T. L., \& Rood, R. 1994, ARA\&A, 32, 191

Woodall, J., Agúndez, M., Markwick-Kemper, A. J., \& Millar, T. J. 2007, A\&A, 466, 1197

Zhang, Q., \& Ho, P. T. P. 1997, ApJ, 488, 241

Zhou, S., Evans, II, N. J., Kömpe, C., \& Walmsley, C. M. 1993, ApJ, 404, 232 